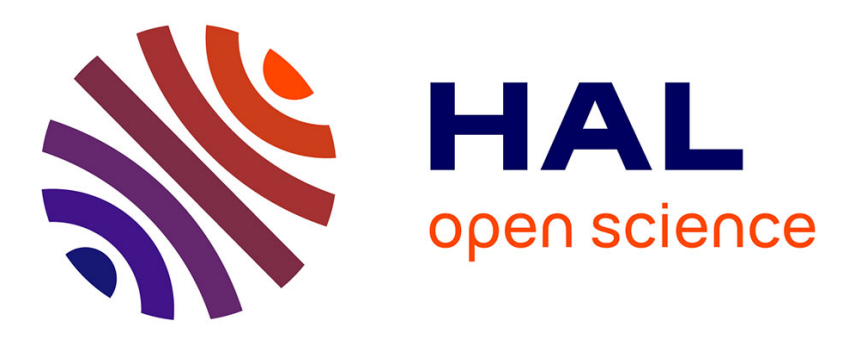

\title{
Action publique et étalement urbain à Rome : une lecture par les services en réseau Hélène Nessi
}

\section{To cite this version:}

Hélène Nessi. Action publique et étalement urbain à Rome: une lecture par les services en réseau. Flux - Cahiers scientifiques internationaux Réseaux et territoires, 2010, 79-80, pp.69-89. 10.3917/flux.079.0069 . hal-00815726

\section{HAL Id: hal-00815726 \\ https://hal-enpc.archives-ouvertes.fr/hal-00815726}

Submitted on 17 Jan 2018

HAL is a multi-disciplinary open access archive for the deposit and dissemination of scientific research documents, whether they are published or not. The documents may come from teaching and research institutions in France or abroad, or from public or private research centers.
L'archive ouverte pluridisciplinaire HAL, est destinée au dépôt et à la diffusion de documents scientifiques de niveau recherche, publiés ou non, émanant des établissements d'enseignement et de recherche français ou étrangers, des laboratoires publics ou privés. 


\title{
Action publique et étalement urbain à Rome: une lecture par les services en réseau*
}

\author{
Hélène Nessi
}

\section{INTRODUCTION. LE DÉSÉQUILIBRE DE LA RÉPARTITION DE LA RENTE URBAINE ENTRE ACTEURS PRIVÉS ET PUBLICS}

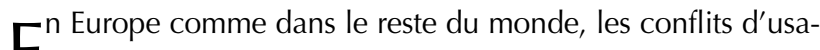
Ege du foncier sont au cœur des politiques d'aménagement visant à contrôler l'extension urbaine dans les secteurs périurbains et à réaliser des services publics, aménager des espaces verts et équiper les zones d'urbanisation. Ces conflits renvoient à la particularité du marché foncier. La rareté des sols et la croissance de leurs prix sont propices à la rente. La rente foncière est définie par David Ricardo, économiste classique du XIXe siècle, comme la " portion du produit de la terre que l'on paie au propriétaire pour avoir le droit d'exploiter les facultés productives et impérissables du sol. » (1992, p. 89, 1 re édition, 1817). La rente se divise en deux composantes: la rente absolue et la rente différentielle (Marx, 1894; Ricardo, 1817). La rente absolue dépend de la capacité d'une aire indépendamment de sa localisation et renvoie à la rareté des sols et à la propriété privée (Malthus, 1820; Ricardo, 1817). La rente différentielle correspond à l'augmentation des valeurs foncières en lien avec la fertilité et la localisation. Elle est donc relative à l'intervention d'acteurs extérieurs, ne dépendant pas de l'effort productif du propriétaire. Après son premier investissement nécessaire pour l'acquisition des sols, le propriétaire n'apporte plus aucuns capitaux. C'est la collectivité qui, au contraire, en ajou- tant des services publics à proximité du terrain, représente le facteur productif (Turner et al., 1996; Franck, 1992).

Parmi les formes de rentes prévues par l'économie, celle qui est la plus importante, tant par ses montants que par ses effets, dans le régime capitaliste, est la rente foncière urbaine (Malthus, 1798; Ricardo, 1817; Walras, 1860). Le revenu net que peut donner la propriété d'un terrain agricole, quelle qu'en soit l'exploitation, n'est pas comparable au revenu obtenu par les propriétaires de sols urbains ou urbanisables (Venuti, 2003). L'accroissement de valeur des sols qui passent de l'exploitation agricole à un usage urbain provient de la collectivité, tant pour ce qui concerne la constructibilité, en délivrant l'autorisation de droits à construire, que pour ce qui concerne les investissements publics, en transformant le sol agricole en terrain équipé à bâtir. Cette rente est perçue par le propriétaire du sol, qui n'a généralement pas contribué aux dépenses d'urbanisation, payées au contraire par la collectivité. Le propriétaire privé s'enrichit pendant que les dépenses publiques s'accroissent.

Le déséquilibre entre les propriétaires fonciers et le secteur public renvoie à un phénomène cumulatif étroitement lié à la rente différentielle. En rendant constructible un terrain, la commune participe une première fois à l'augmentation de sa valeur, faisant passer la rente agricole à la rente urbaine. La commune doit alors urbaniser la zone et y réaliser des équipements publics et, pour cela, acquérir des terrains. Via la réalisation de 
ces équipements, l'autorité publique participe une seconde fois à la croissance de la rente urbaine des terrains privés. La collectivité publique se confronte donc à deux difficultés objectives: d'une part, elle augmente la rente urbaine des terrains privés en y apportant les équipements et les services publics; d'autre part, elle fait face à la difficulté d'acquisition de surfaces disponibles pour les services compte tenu de leur prix élevé du fait même de son intervention. Le développement des opérations privées sur le territoire accroît les dépenses publiques qui alimentent la rente pour les propriétaires fonciers.

Face à ce déséquilibre, les acteurs publics ont tenté de mettre en place des procédures de contrôle du marché: via la réglementation urbanistique, la collectivité peut modifier la répartition de la rente urbaine. En termes financiers, une politique foncière peut infléchir la redistribution inéquitable générée spontanément par le fonctionnement des marchés (Ricardo, 1817; Von Thünen, 1826; Marshall, 1890; Marx, 1894; Alonso, 1968). Plus récemment en Europe, dans un contexte de souci de réduction de leurs dépenses ou d'accroissement de leurs recettes, les pouvoirs publics ont mis en place des procédures innovantes de négociation entre le secteur public et le secteur privé. Quelle est la meilleure façon pour la collectivité de récupérer ces plus-values foncières comme nouvelles sources de financement des réseaux d'infrastructure et d'équipements publics? Comment faire évoluer la prise en charge des dépenses de viabilisation et d'équipements (Comby, 2003; Renard, 2007)? Deux stratégies d'actions se dégagent: dans les pays du Nord de l'Europe, des politiques publiques de maîtrise foncière ont permis une bonne régulation de l'urbanisation et une quasi-absence de spéculation foncière; dans les pays du sud, face à un fort pouvoir des propriétaires fonciers, et donc à une forte spéculation foncière, la collectivité doit passer par des politiques de négociation (Renard, 2007).

La littérature sur la maîtrise de la rente foncière urbaine par le secteur public fait état de trois voies possibles et complémentaires: (a) la voie fiscale, (b) la voie de l'expropriation et (c) la voie des droits de construction négociables associés la répartition des coûts d'urbanisation.

(a) La voie fiscale s'inscrit dans le courant réformiste. Les réformistes critiquent le capitalisme sans toutefois remettre en cause son existence; ils cherchent des outils pour le réformer afin d'en maîtriser les inconvénients. À ce titre, Mill (1848) n'interdit pas l'existence de la pro- priété privée mais s'interroge sur le bien-fondé de la rétribution d'une rente au propriétaire. Dans ce cadre, il préconise l'appropriation de la rente foncière par l'État via une taxation des plus-values foncières. Une autre procédure consiste à confisquer la rente par le biais d'un impôt foncier unique (George, 1880). Cette stratégie a d'ailleurs été appliquée dans les années cinquante dans certains pays comme la Nouvelle-Zélande, I'Afrique du Sud, le Danemark et, aux USA, à Pittsburgh.

(b) La procédure de maîtrise du foncier la plus classiquement utilisée par la puissance publique demeure l'expropriation, qui permet de sortir le sol du marché via le rachat des terres par la puissance publique (Proudhou, 1840; Walras, 1896; Lénine, 1907); mais cette procédure génère des risques juridiques et des coûts administratifs très élevés, ce qui explique la faible propension des collectivités locales à y recourir. En outre, payer le prix du marché au propriétaire est souvent difficile à soutenir sur le plan financier, sauf à avoir anticipé les hausses de prix du foncier liés à l'urbanisation en se dotant au préalable de réserves foncières.

(c) Enfin, au début des années 1990, des procédures instaurant des droits de construction négociables et une répartition des coûts d'urbanisation ont fait l'objet de discussions académiques et d'expériences administratives limitées. Depuis, l'intérêt des scientifiques, des politiques et des technico-professionnels à l'égard de cet outil s'est considérablement accru. Avec d'importantes différences, des politiques d'acquisition du foncier et de redistribution des coûts de l'urbanisation, moyennant le transfert des droits, ont été expérimentées en France et en NouvelleZélande (Renard, 1998), en Espagne (Garcia-Bellido, 1997), en Italie (Lanotte, Rossi, 1995; Stanghellini, 1997; Micelli, 2002) et aux États-Unis (Hagman, Misczynski, 1978; Johnston, Madison, 1997; Jacobs, 1997, 2004; Pruetz, 1996). Dans le panorama européen, trois logiques de redistribution des coûts de I'urbanisation existent. (a) Les propriétaires fonciers ont à leur charge l'urbanisation car les équipements publics donnent de la valeur à leurs terrains. (b) Les charges d'urbanisation reviennent aux constructeurs, car les nouvelles opérations rendent nécessaire la réalisation des nouveaux équipements. (c) Les contribuables assument le coût de l'urbanisation car les équipements sont publics, via la fiscalité locale. 
La rente urbaine est donc une notion clef pour comprendre le déséquilibre existant entre les propriétaires fonciers privés et le secteur public. Depuis les années 1990, les procédures de négociation de droit de construction sont des modalités particulières de la maîtrise de la répartition de la rente urbaine entre acteurs privés et publics. Ce type de négociations sera analysé à partir du cas romain. Tout d'abord, nous montrerons comment les politiques issues de l'analyse classique en termes d'appropriation de la rente foncière $s^{\prime}$ avèrent insuffisantes pour résoudre les conflits liés à la rente différentielle et les problèmes de sous-équipement de la ville. Nous nous attacherons ensuite aux politiques de négociation en focalisant notre analyse sur les modalités de financement des infrastructures en réseau et des services collectifs. En dépassant les débats idéologiques, cette analyse vise à évaluer les capacités et modalités d'action concrète de la municipalité en matière de planification urbaine et de redistribution de la rente urbaine.

\section{ROME: DU FONCIER AU RÉSEAU}

L'Italie et sa capitale constituent un terrain original pour I'observation du foncier, véritable enjeu des politiques urbaines concernant l'étalement urbain. Le déséquilibre préexistant entre secteurs privé et public compromet l'acquisition des terrains pour la réalisation d'équipement publics (1). Le Piano regolatore generale (PRG), instrument complexe et hégémonique de la pratique planificatrice italienne, issu de la vieille loi sur l'urbanisme de 1942, est aujourd'hui largement critiqué pour son incapacité à encadrer les évolutions urbaines et à contrôler la rente foncière (Venuti, 2002 et 2003; Berdini, 2006; Gibelli, Salzano, 2006). Face à cette constatation, I'Institut national d'urbanisme italien a développé à son tour des outils de négociation et considère les droits négociables comme un atout de sa proposition de réforme (2) de l'urbanisme, visant à régler la disparité juridique entre droits publics et privés. À la fin des années 1990, lors de la mise en œuvre des droits négociables dans les plans d'urbanisme de nombreuses communes, grandes et moyennes, certains chercheurs se sont intéressés à cette nouvelle pratique (Stanghellini, 1993; Barbieri, Oliva, 1995; Barbieri, 1998; Fusco-Girard, 1997; Pompei, 1998; Micelli, 1999, 2003), se limitant souvent à des débats idéologiques opposant les réformistes et les contre-réformistes. Alors que Rome constitue un des laboratoires urbanistiques de ce système de négociation des années 1990-2000, aucun de ces travaux de recherche n'a jamais abordé le cas romain.

\section{Rome: un immense territoire aux mains de quelques grandes familles}

$C^{\prime}$ est à divers titres que Rome constitue un cas intéressant. L'histoire urbaine est marquée par un déséquilibre important entre une Commune dépourvue de patrimoine foncier et de moyens financiers, et quelques grandes familles et sociétés immobilières propriétaires de $90 \%$ du territoire. À Rome, la rente urbaine présente les caractéristiques d'une rente d'oligopole: ces grandes familles, à la fois propriétaires du foncier et constructeurs, sont en mesure de s'approprier la rente urbaine. " Les prix de toutes les surfaces centrales intermédiaires et périphériques se maintiennent à des niveaux très élevés, en constante augmentation. Le marché des terrains à bâtir est un marché imparfait dans lequel I'offre se développe à des prix qui ne résultent pas du jeu de l'offre et de la demande, au grand dam de la collectivité. »(Venuti, 2003, p. 23). La crise des années 1990 et surtout la reprise vigoureuse qui a suivi depuis 1996 ont renforcé les positions dominantes de ces acteurs.

Si elle est confrontée à l'étalement urbain comme la majorité des villes européennes, la commune de Rome offre un terrain original pour l'étude de ce phénomène (Figures 1 et 2). La capitale italienne, 12 fois Paris intra muros, est la commune la plus étendue d'Europe. Avec, en 2006, une superficie d'environ 129000 hectares dont seulement 55500 ha urbanisés (ce qui laisse $57 \%$ de terrains agricoles), elle est directement concernée par la dispersion urbaine qui se développe très largement au sein même de ses limites administratives. Un des phénomènes majeurs à l'origine de cette expansion urbaine est celui de I'abusivismo (Della Seta et Della Seta, 1988) concernant I'urbanisation de 11000 hectares (800000 habitants) (3) du territoire romain (Berdini, 2006) (voir encadré). Les nombreux quartiers abusifs qui voient le jour à Rome présentent des carences en termes $d^{\prime}$ infrastructures primaires (4) et secondaires (5). L'abusivismo produit une périphérie diffuse, anarchique, sans infrastructure ni service (Rossi, 1975). Ajouté au phénomène de I'abusivismo, le déséquilibre entre la Commune, dépourvue de patrimoine foncier et de moyens financiers, et quelques grandes familles et sociétés immobilières marque I'histoire urbaine de la "Troisième Rome » (6). Tout un courant historiographique, proche du marxisme, a montré que la ville s'est construite en grande majorité en dehors des cadres de la planification urbaine, selon un système de conventions entre la mairie et les promoteurs (7). En particulier, l'administration démocrate-chrétienne, étroitement liée aux intérêts privés, a gouverné la ville sans 
Figure 1. Évolution de la consommation des sols et de la démographie

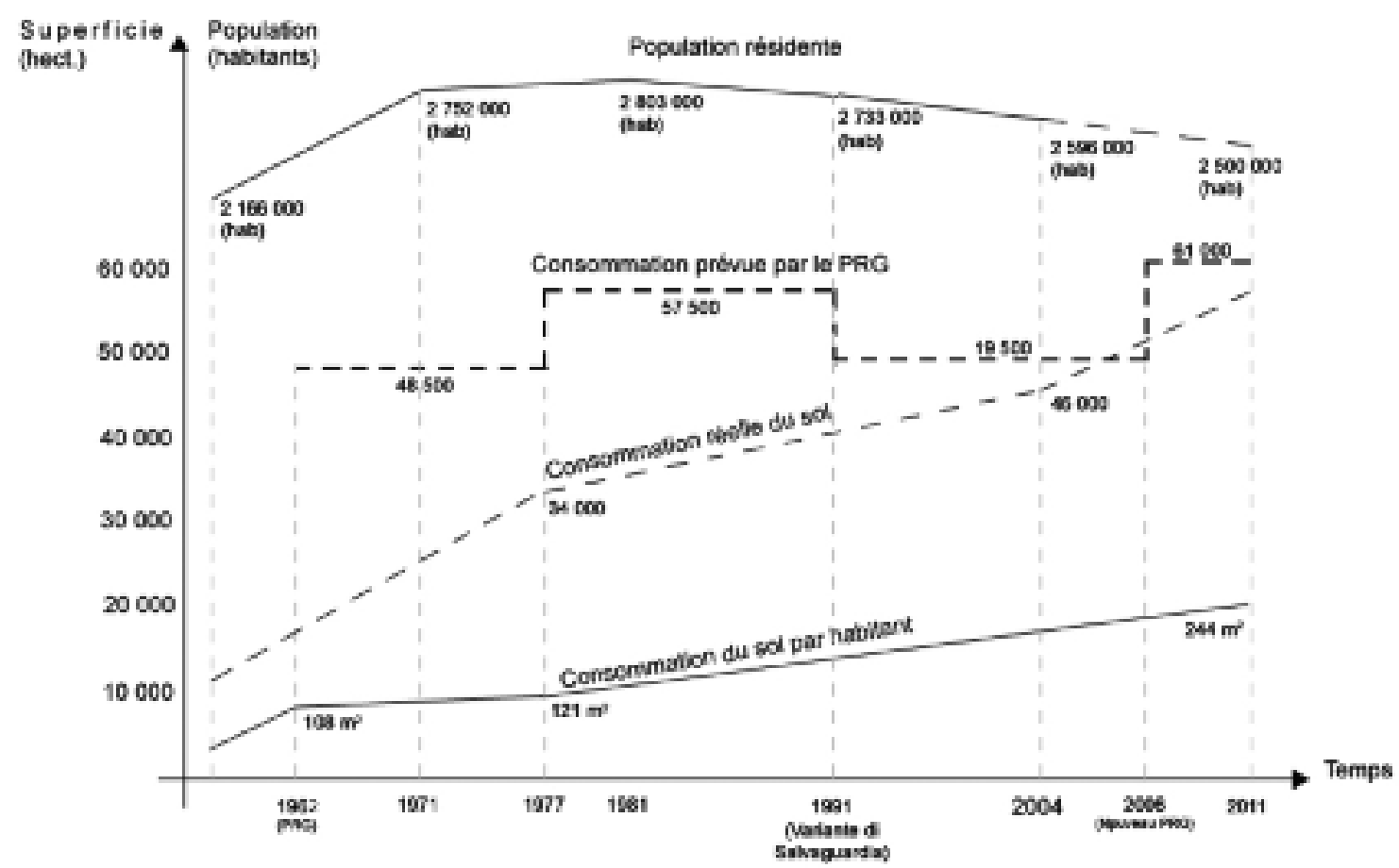

Source: BERDINI P., 2005, La cancelianione dolla campagna romans, in * Na sprawl x, MCC. Gibeli e E.Salzano, Ninez Editrice

Ce graphique exprime revolution de la consommation des sols au regard de l'évolution démographique de 1962 à 2011. A la consommation des sols prévue par le plan régulateur de 1965, soit 48500 ha, s'ajoute en 1977 les 9 CCO ha provoques en partie par l'assainissement et la recuperation des zones abusives et par les variantes. En 1991, la diminution de la superficie constructible, due a la variante de sauvegarde compense quasi entierement les augmentations précédentes. Les 11500 ha prévus dans le nouveau plan régulateur de 2006 (normalement prescriptif pour les 5 prochaines années) ne font que prendre en compte la croissance urbaine de 2003 a 2006 deja réalisee. L'indice de consommation des sols en 2011 est le double de celul prévu en 1965. Alors que lexpansion de 1962 à 1977 était liée à une croissance démographique, les prévisions urbaines du nouveau plan sont expliquées exclusivement par des dynamiques économiques". On relove en effet de 1991 à 2011 une perte de 178000 habitants". "A Rame ie ierritoire devient une variable éconamigue laisste aux mains de la speculanion" (Berdini, 2006)

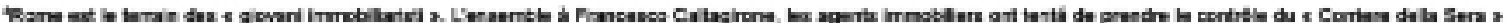

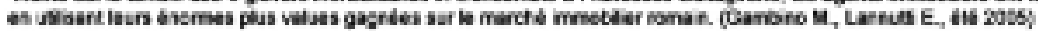

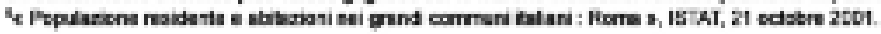

les moyens, ni d'ailleurs la volonté de stopper l'extension. Mais même les tentatives de la gauche, attaquant de front la question de la rente urbaine, n'ont pas résolu les problèmes de régulations urbaines.

\section{Les échecs de la planification romaine: l'intérêt d'une lecture par les services en réseaux}

L'administration de gauche, qui a gouverné la ville de 1976 à 1985, s'est engagée dans une lutte acharnée mais souvent vaine 


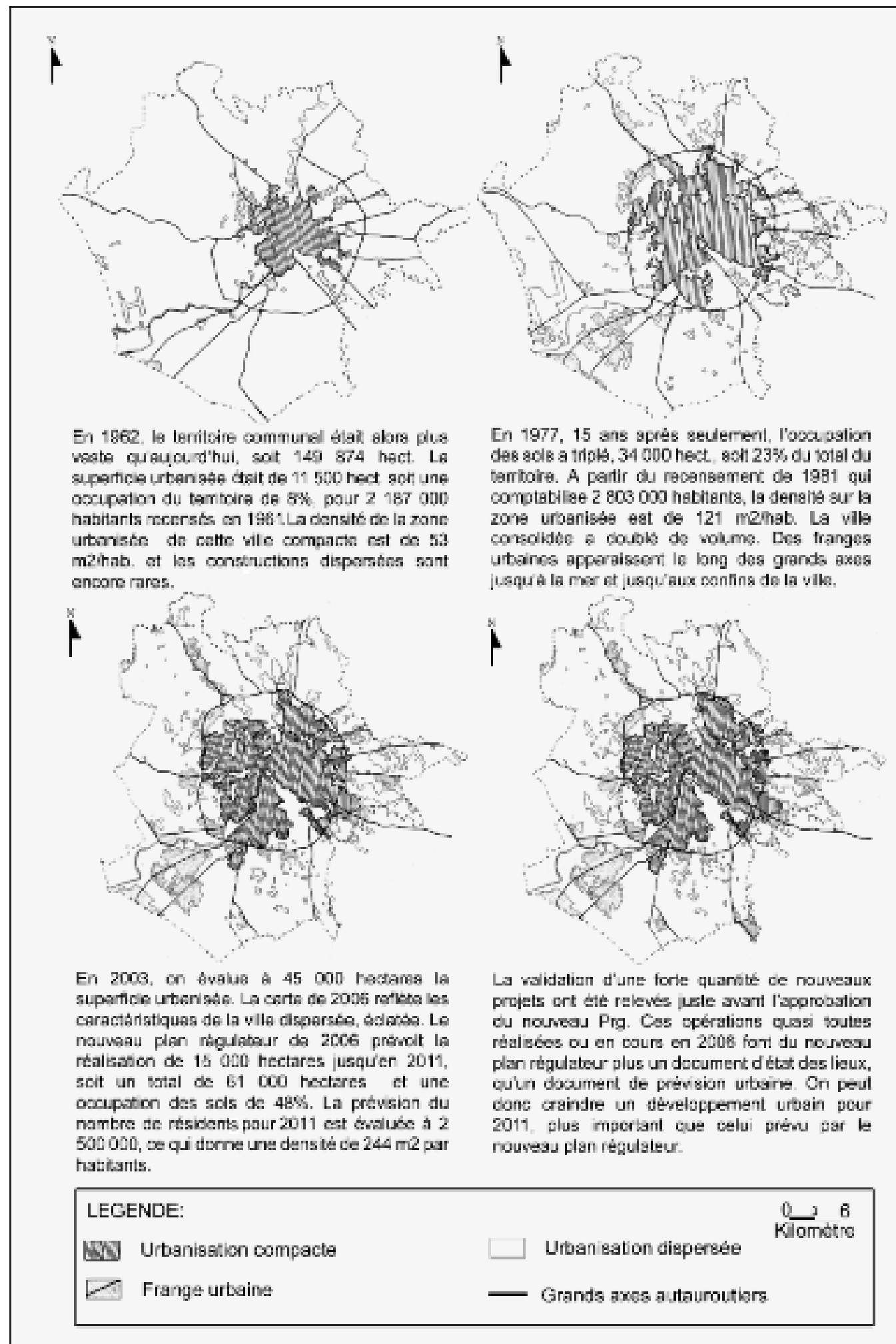

Figure 2. Développement urbain

Source: réalisation de l'auteure 


\section{$\mathbf{L}^{\prime}$ « abusivismo »}

Le phénomène de l'abusivismo apparaît sous le régime fasciste entre 1925 et 1939. Pour laisser place aux grands travaux de modernisation de la capitale romaine, des quartiers populaires situés en plein centre de Rome sont entièrement rasés. Promues par la politique fasciste, les premières constructions abusives voient le jour en toute légalité à la fin des années 1920 afin de reloger en périphérie les résidents expulsés des quartiers centraux.

Dans les deux décennies d'après guerre, la ville, en pleine reconstruction, est sujette à une forte croissance urbaine. Pour répondre à la demande de main-d'œuvre, cette croissance rapide s'accompagne d'une grande vague d'immigration provenant du sud de I'Italie. En l'absence de logement pour leurs familles, les ouvriers acquièrent des terrains et bâtissent sans autorisation. Des noyaux résidentiels illégaux apparaissent sous le nom d' « abusivismo de nécessité », (Santuccio, Giancola, Piccioto, 1976), 44 zones abusives soit 3800 hectares sont recensées dans le plan régulateur de 1962.

À partir des années 1970, les constructions abusives s'installent dans les grands espaces vides, résidus des piani di zona (8) (plan de zone) inachevés, ainsi qu'en périphérie sur l'agro romano le long des axes routiers principaux. Non viabilisés, ces quartiers engendrent des problèmes de précarité et d'insalubrité. C'est seulement en 1985 que ces habitations sont reconnues juridiquement: 85 noyaux abusifs, soit 4700 hectares sont alors recensés (1re loi sur le condono edilizio édictée par le gouvernement Craxi).

Dans les années 1990, un nouveau type de construction illégale, n'ayant plus de caractère de nécessité, apparaît: « l'abusivismo de luxe », implanté sur des aires prisées au bord de la côte ou dans des parcs (Bossi, 2003). Le gouvernement Berlusconi instaure à son tour, en 1994 puis en 2003, de nouvelles procédures de légalisation des constructions illégales. Ce sont alors 55 noyaux illégaux, soit 2400 hectares, qui sont régularisés.

Cette pratique illicite subsiste encore aujourd'hui mais dans des proportions nettement moins importantes. En 2003, on estime à 11000 hectares l'étendue du phénomène, concernant 800000 habitants.

contre la spéculation foncière et immobilière - et dont les effets sur l'abusivismo vont être limités. La commune adopte progressivement une planification visant à limiter le développement des occupations illégales. Ainsi, dans les années 1970, la commune programme la réalisation de logements sociaux avec les piani di zona. Cette programmation s'inscrit dans une logique purement quantitative des interventions (mesurées en $\mathrm{m}^{2}$ ou en $\mathrm{m}^{3}$ construits), prévoyant des projets résidentiels monumentaux, accompagnés d'espaces surdimensionnés pour accueillir les équipements et services publics. Mais ces équipements, prévus par les plans de zones sur des emprises réservées, ne seront jamais réalisés par manque de volonté politique et de moyens financiers. En définitive, au lieu de limiter le phénomène de l'abusivismo, cette politique favorise sa propagation en permettant l'implantation de nouvelles constructions sur ces grands espaces vides. À la périphérie abusive antérieure déjà sous-équipée, s'ajoutent ces nouveaux quartiers également privés de connexions aux services urbains en transport et en services publics.
Les planificateurs de la municipalité de gauche, s'appuyant sur des travaux $d^{\prime}$ inspiration marxiste, se sont focalisés sur la rente urbaine et ont été incapables de mettre en place un modèle de régulation des sols leur permettant de maîtriser la rente foncière. Si la question de la rente foncière est tout à fait pertinente comme catégorie d'analyse, elle s'avère insuffisante sur le plan de l'action: en effet, la posture sans concession consistant à rejeter tout compromis avec les propriétaires en termes de partage de la rente foncière s'est révélée contre-productive. À aucun moment, les planificateurs de l'administration de gauche ne se sont intéressés à la situation critique du territoire en termes d'équipements publics et d'infrastructures, ni n'ont pris en compte le droit des citoyens à être connectés aux services. Pour eux, I'appropriation de la rente urbaine par quelques grands propriétaires terriens était le symbole d'une pathologie romaine (Venuti, 2003) dans laquelle la valeur des terrains n'est pas liée à la rareté relative des sols mais à la coalition tacite entre les propriétaires, les actionnaires de la société de transports collectifs et les édiles locaux (Delpirou, 2009). 
De plus, le déséquilibre préexistant entre secteurs privé et public en termes de droit sur les sols, lié au contexte de monopole du foncier par quelques grandes familles romaines, a été exacerbé suite à la décision institutionnelle de 1984 qui a réduit à cinq ans la durée de validité des droits publics d'expropriation: à la différence des droits privés, les droits de la commune se trouvent ainsi limités dans la durée. Pour pouvoir conserver ses droits d'expropriation au-delà du terme prévu par la loi, la municipalité doit payer, année après année, une véritable " indemnité de servitude » aux propriétaires fonciers; par manque de ressources financières, elle est donc conduite progressivement à renoncer à ces droits et l'acquisition des terrains pour la réalisation d'équipements publics s'en trouve compromise. Les instruments reposant sur le contrôle de la rente foncière s'avèrent donc incapables de traiter les problèmes de sous-équipement de la périphérie. De fait, les instruments de planification et, d'abord, le Piano Regolatore Generale (PRG) sont aujourd'hui largement critiqués pour leur incapacité à contrôler la rente foncière, mais, surtout, pour celle à encadrer les évolutions urbaines.

Le contrôle de la croissance urbaine et le raccordement aux réseaux (voirie, transports collectifs, électricité, eau, assainissement) des zones abusives sont donc les deux grandes difficultés de la planification romaine depuis vingt ans. Dans un contexte où l'approche réglementaire des problèmes d'usage des sols n'est que partiellement opérante (du fait des pratiques répandues de contournement du plan), le pouvoir de la municipalité romaine, qui dispose de ressources foncières et financières restreintes, est limité face à la dispersion de l'urbanisation sur son territoire. Pourtant, confrontée aux défis de la croissance urbaine et de la légalisation progressive des quartiers illégaux, la commune a développé des mécanismes de financement des services urbains pour les travaux de requalification des zones abusives et pour les nouvelles opérations privées de constructions résidentielles. Ces négociations empruntent des voix originales reposant sur la prise en charge des coûts d'urbanisation par le secteur privé. D'une part, les conventions sont bien une manière de redistribuer la rente foncière au bénéfice de la collectivité, des équipements et des services publics; d'autre part, elles $\mathrm{n}^{\prime}$ influent pas exclusivement sur le partage de la rente, mais aussi sur la localisation, la nature et la forme des projets, la qualité des formes urbaines produites, celle des équipements et services publics, etc. Une entrée par l'analyse des réseaux permettra d'apporter un éclairage sur les outils mis en place par
I'autorité publique pour maîtriser ou limiter la rente urbaine et d'évaluer ainsi l'efficacité et les effets sur l'urbanisme de ces outils.

\section{La négociation privé/public: la base des procédures d'adaptation communale aux législations nationales}

Deux textes importants marquent, au niveau national, le domaine de l'urbanisme en Italie dans la période récente: la loi Bucalossi de 1977 concerne les constructions privées légales et la loi sur le condono edilizio (amnistie immobilière) de 1985 les constructions illégales. Ces lois sont censées apporter des réponses aux problèmes d'insalubrité des périphéries urbaines et de financement des équipements primaires et secondaires et aux difficultés résultant de l'ampleur de l'urbanisation sur les territoires.

La loi Bucalossi modifie sensiblement le cadre juridique des contributions aux équipements. Alors que, jusqu'en 1977, pour réaliser légalement un projet, les propriétaires privés n'avaient rien à verser à la collectivité publique lors du dépôt de permis de construire, cette loi établit le principe que le droit de construire est une concession de la puissance publique accordée aux pétitionnaires. En conséquence, ce droit est payant. Le montant de la Concessione edilizia comporte deux éléments: la participation au coût des équipements primaires et secondaires et le droit à construire proprement dit.

La loi sur le condono edilizio (amnistie immobilière) prévoit la régularisation des constructions illégales et la requalification de ces zones abusives par la municipalité. Dans le cadre de cette loi, les propriétaires versent à l'État le montant d'une amende et à la commune les honoraires des droits de construction, établis en fonction du nombre de $\mathrm{m}^{2}$ et de $\mathrm{m}^{3}$ des constructions et du type d'infraction (Lenzini, Ricardo, Ruggero, Giannini, 2004). En contrepartie de leur régularisation, les terrains doivent désormais être équipés par la commune en services urbains de base. Le premier condono edilizio date de 1985; il est suivi par deux autres condoni edilizi sous le gouvernement Berlusconi en 1994 et 2003.

Ces changements pénalisent la municipalité. La loi Bucalossi, même si elle impose une contribution au secteur privé, laisse une nouvelle marge de manœuvre à son opportunisme. Quant aux mesures de requalification des zones abusives dans le cadre des condoni edilizi, elles sont lentes et ne résolvent pas le manque de moyen de la commune. Le gouvernement romain 
D'un point de vue méthodologique, cette recherche repose sur trois éléments principaux: (a) une analyse contextuelle pour chaque opération immobilière, (b) l'analyse de documents contractuels nécessaires à la réalisation d'une opération et (c) la réalisation d'entretiens auprès d'hommes politiques, de techniciens, d'urbanistes, de chercheurs et de membres d'associations environnementales.

(a) L'analyse contextuelle des projets a permis de relever l'état réel des opérations.

(b) L'acquisition et l'analyse des documents contractuels des projets étudiés dits d'« accord de programme ", a permis la connaissance des accords établis entre la commune et les constructeurs aussi bien dans la programmation du projet que dans sa répartition financière. Une confrontation entre les analyses contextuelles et les documents contractuels a permis de pointer les décalages entre la programmation des projets et leur réelle réalisation.

(c) Des entretiens ont été réalisés de 2007 à 2009 avec des responsables d'institutions publiques afin d'identifier les enjeux du territoire: à l'échelle des arrondissements IV, V, VIII et XIX avec les responsables de l'urbanisme et des travaux publics (7 entretiens), à l'échelle de la commune romaine avec les responsables de l'urbanisme (2), des travaux publics (2), de la mobilité (3), de la programmation et de la planification territoriale (1) et de la périphérie/Condono Edilizio (5), à l'échelle de la province avec le responsable de la planification urbaine (1), à l'échelle de la région Lazio avec les responsables de l'urbanisme (1), des travaux publics (1), de la mobilité (2) et de l'environnement (3). Enfin, des entretiens avec des techniciens de la commune ACEA (entreprise responsable des réseaux d'électricité et d'eau) (5), ATAC (3) et FFSS (1) (entreprises responsables des réseaux de transports en commun), AMA (entreprise responsable des déchets) (2) et Eni (entreprise responsable du réseau de gaz) (1), des urbanistes (3), des chercheurs dans le domaine de la planification et les réseaux d'infrastructure de l'université de la Sapienza (3) et de l'uiversité de Roma III (1) et des membres des associations environnementales: Legambiente (2) et Italia Nostra (2) et une association d'habitants (1) ont permis de préciser le contexte dans lequel les opérations ont été mises en œuvre. L'obtention de données et de renseignements complémentaires issus de ces entretiens a permis d'interpréter au mieux les discours politiques et les dynamiques urbaines observées; et d'identifier les décalages entre le discours politique et la réelle répartition des coûts de l'étalement urbain. Au total, 52 entretiens ont été menés.

de gauche avait d'ailleurs protesté à l'époque contre les rétributions encaissées par l'État à la faveur des trois lois successives alors que c'est la commune qui doit supporter les frais de la requalification promise par la loi, frais plus importants que le montant des honoraires qu'elle perçoit. En outre, le gouvernement de gauche de Rome avait dénoncé, dans le condono edilizio mené par l'État, une manœuvre politique de l'ancien chef du gouvernement de droite, $\mathrm{S}$. Berlusconi, pour attirer de nouveaux électeurs dans son camp.

En 1993, l'élection à Rome d'une municipalité progressiste se traduit, dans le champ de l'urbanisme, par la mise au point d'une stratégie d'aménagement originale, conçue comme alternative aux politiques antérieures. La recherche de compromis avec les propriétaires a été au cœur de la politique urbaine des maires de centre gauche, Rutelli (1993-2000) et Veltroni (20012008), précédant et accompagnant les longues procédures d'élaboration et d'approbation du nouveau plan régulateur. Pour faire face aux difficultés de financement des coûts de la requalification non résolues par les lois nationales, la commune met en place de nouvelles procédures avec, pour les constructions privées légales, la Convenzione urbanistica en 1993, et, pour les constructions illégales, la procédure de requalification d'autorecupero des zones abusives, à partir de 1994. « La fiscalité immobilière, notamment, doit être considérée comme complémentaire aux droits négociables dans le prélèvement de parts de rente urbaine » (Micelli, 1999; voir aussi : Camagni, 1992; Micelli, 2002, 2003). Les innovations romaines, emblèmes d'un urbanisme qualifié de "réformiste », ont engendré de violentes polémiques qui ont conduit à la division du camp progressiste entre réformistes et " contre-réformistes » (Venuti, 1991, 2003), sur fond de rivalités institutionnelles (Stanghellini, 1993; Barbieri et Oliva, 1995; FuscoGirard, 1997, Pompei, 1998) et de querelles idéologiques. Les contre-réformistes, $\mathrm{d}^{\prime}$ inspiration marxiste, accusent la municipalité de se compromettre avec le secteur privé et dénoncent I'accord sans limite de nouveaux droits de construction toujours 
plus loin du centre. En se focalisant sur le mécanisme structurel de la rente foncière et de la rente urbaine, ils s'interdisent de se demander si ces négociations avec le secteur privé peuvent, éventuellement, apporter des avantages et des marges de manœuvre à la collectivité.

Pour sortir de ces débats idéologiques, nous avons mené un travail $d^{\prime}$ analyse comparant la répartition privé/public des coûts d'urbanisation selon les procédures utilisées (nationales, municipales). Nous avons choisi de limiter notre analyse aux charges d'investissement qui constituent la dépense principale (même $s^{\prime}$ il conviendrait en toute rigueur d'ajouter les coûts d'exploitation et de maintenance). Ce qui nous intéressera ici, davantage que le niveau des coûts, ce sont les mécanismes de répartition de ces coûts entre les différents acteurs. Pour décrire ces

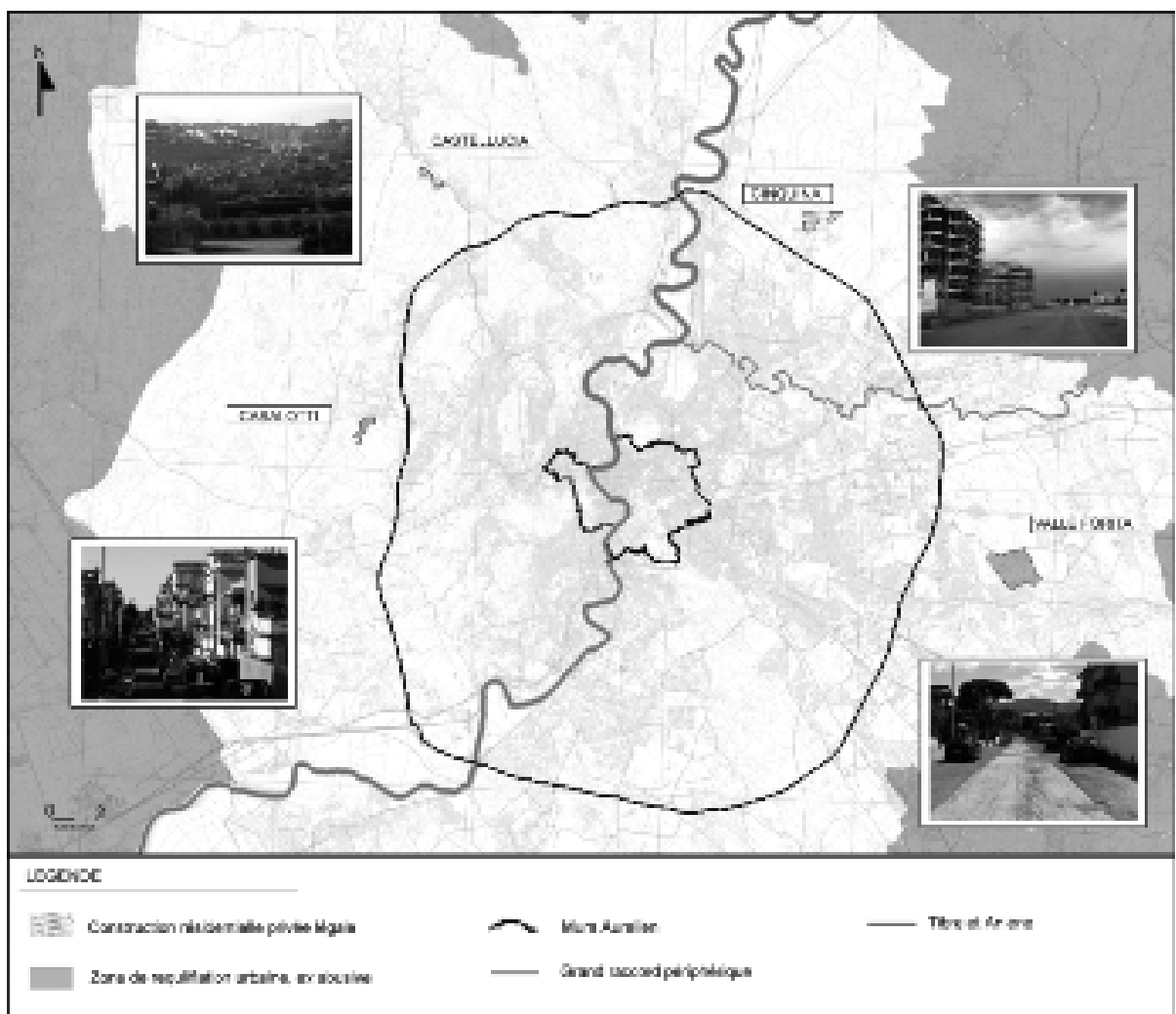

Figure 3. Situation des projets. Source: réalisation de l'auteure mécanismes, deux cas représentatifs des dynamiques urbaines à Rome seront étudiés en détail : les constructions illégales et les constructions légales. L'analyse des mécanismes de financement des lois nationales et de leur adaptation par les nouvelles procédures communales en ce qui concerne ces deux types de construction suggère que la commune ne sort pas nécessairement perdante de ces négociations. Les modalités concrètes de mise en œuvre de ces dispositifs sont étudiées ci-dessous dans quatre études de cas d'urbanisation périphérique. Nous présenterons d'abord comment la commune, dans le cadre de la requalification des zones abusives, parvient à accélérer l'équipement de la périphérie en faisant contribuer les propriétaires privés. Dans un deuxième temps, nous montrerons comment la municipalité, en agissant sur des opérations de constructions privées légales, parvient à réduire l'opportunisme du secteur privé grâce à un nouveau type de contrat basé sur la négociation public-privé. Ces études de cas présentent une certaine analogie avec l'approche mise en œuvre par les acteurs euxmêmes dans d'autres contextes: il semble en effet que, souvent, les pouvoirs publics cherchent moins à connaître précisément le niveau des coûts qu'à en définir les modalités de répartition et à maîtriser la part leur revenant. Ainsi, bien que très encadré par les textes réglementaires, le financement des infrastructures résulte aussi d'arbitrages politiques au sein même des communes (Jaglin et al., 2008).

\section{L'INTERVENTION DU SECTEUR PRIVÉ POUR ACCÉLÉRER LA REQUALIFICATION DES ZONES ABUSIVES}

Les procédures liées à la régularisation des zones abusives seront traitées à partir de deux études de cas choisies pour illustrer, d'une part, le cadre de la première régularisation nationale, le condono edilizio de 1985, avec l'exemple de Valle Fiorita et, d'autre part, le dispositif dit d'autorecupero établi par la municipalité en 1994 lors du tournant réformiste, avec I'exemple de Casalotti. Ces deux zones sont situées en extrême périphérie au-delà du grand raccord annulaire (Figure 3), I'une étant desservie par le réseau ferroviaire et l'autre non; leur urbanisation est issue du phénomène de l'abusivismo. 


\section{Valle Fiorita ou les lenteurs de la requalification des zones abusives}

Le cas de Valle Fiorita illustre les difficultés rencontrées par la commune lors de la réalisation des travaux menés dans le cadre du premier condono edilizio. Les constructions concernées par ce premier condono edilizio sont les édifices réalisés sans permis sur des aires non constructibles ainsi que les restructurations, agrandissements, travaux de façades sans autorisation, réalisés avant 1983. Comme expliqué auparavant, les propriétaires constructeurs versent à l'État le montant d'une amende et à la commune les honoraires de droit de construction. En contrepartie de la régularisation, la loi contraint les municipalités à s'engager à offrir aux citadins concernés un accès équitable aux services urbains essentiels et à améliorer leur condition sanitaire. Ces aires abusives régularisées, dites zones « $\mathrm{O}$ » (9) (Figure 4), sont délimitées par les « piani particolareggiati » (plans particuliers): plans déterminant des zones urbaines à requalifier et intégrés comme « variantes » au plan régulateur de 1962. Les grandes interventions entreprises par la commune pour améliorer ces zones « $\mathrm{O}$ » reposent essentiellement sur l'apport de trois types de services en réseau (10): le réseau d'assainissement (avec la disparition des fosses septiques au profit $d^{\prime}$ 'un système d'assainissement collectif et la réalisation de stations d'épuration collectives pour des groupements de constructions abusives), la voirie et l'éclairage public.

La zone « $\mathrm{O}$ » Valle Fiorita située à l'Est de la ville, au-delà du boulevard périphérique, s'étend sur 117,13 ha et se compose d'environ 750 résidences et 15380 habitants, soit une densité de 130 hab/ha (Figure 3). La régularisation de ces constructions abusives a permis, dans les années 1980, de faire un état des lieux des besoins en services. Mais, c'est seulement en 2005 que le piano particolareggiato est établi. La priorité du

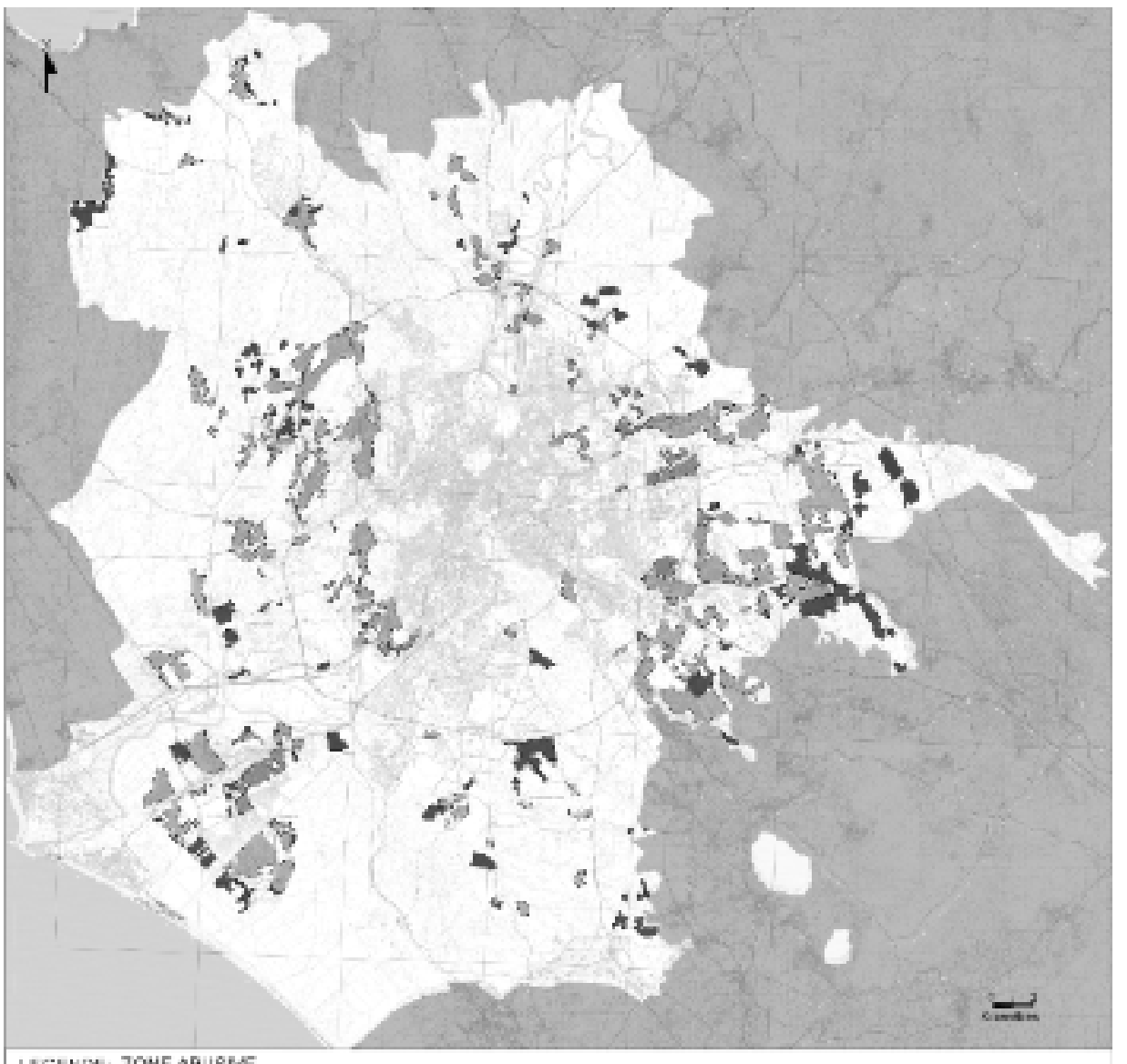
LLGLNDL: 2ONE ABJSME
ZDNE DE TOPUNMI

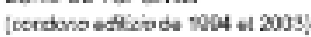
plan est la rationalisation des réseaux au sein même de cette zone et leur connexion aux réseaux principaux des quartiers voisins mais il $\mathrm{s}^{\prime}$ agit aussi de construire un centre religieux et culturel, un marché, un espace vert équipé et un parc. Le tableau 1 restitue la répartition des dépenses de la requalification de la zone (Tableau 1).

À Valle Fiorita, comme ailleurs, la municipalité est confrontée aux difficultés techniques d'insertion des infrastructures dans un tissu urbain déjà existant, qu'il s'agisse de l'évacuation des eaux en galerie souterraine, de l'élargissement des voies routières ou de la récupération des eaux de pluie. En surchargeant les réseaux principaux, alors que ceuxci sont déjà saturés, ces extensions complexifient le système et en réduisent les performances. En outre, malgré la mise en place de ce mécanisme, la commune de Rome ne résout pas son problème de manque de terrain. À Valle Fiorita, le coût de l'expropriation des espaces nécessaires
DONE "O icundero natriv to 1665]

Figure 4. Zones abusives 
pour l'implantation des services publics (centre culturel, centre religieux ou parcs) et pour l'élargissement de la voirie afin $d^{\prime}$ installer les infrastructures adéquates et faciliter la circulation dans les quartiers correspond à environ $10 \%$ des dépenses totales. Le coût de la requalification de la zone prend des proportions démesurées (11) puisque la ville, en plus de financer les réseaux $d^{\prime}$ infrastructure de service urbain, doit payer l'expropriation. En outre, la lenteur des négociations du fait des procédures admi-

\begin{tabular}{|c|c|c|}
\hline \multicolumn{3}{|c|}{$\begin{array}{c}\text { Tableau 1. Projet de Requalification zone « } O \text { " Valle Fiorita } \\
\text { (117ha, } 15 \text { 380habitants) }\end{array}$} \\
\hline Projet de Requalification & $\begin{array}{l}\text { Coût de la requalification } \\
\text { pour la commune de Rome } \\
\text { (millions d'euros) }\end{array}$ & Programme de requalification \\
\hline Services primaires & 16,6 & $\begin{array}{c}\text { Réseau d'assainissement, micro- } \\
\text { stations d'épuration, éclairage } \\
\text { public, voirie et parking }\end{array}$ \\
\hline Services secondaires & 57,7 & $\begin{array}{l}\text { Centre religieux et culturel, } \\
\text { marché, espace vert équipé, } \\
\text { équipement sportif et parc }\end{array}$ \\
\hline Expropriation des sols & 9,3 & $\begin{array}{c}22,5 \text { ha pour destination } \\
\text { publique } 12,46 \text { ha implantation } \\
\text { du service en réseau }\end{array}$ \\
\hline Montant du projet & 83,6 & \\
\hline $\begin{array}{l}\text { Montant perçu par les hono- } \\
\text { raires de régularisation }\end{array}$ & 5,25 & \\
\hline $\begin{array}{l}\text { Dépense effective de la com- } \\
\text { mune }\end{array}$ & 78,35 & \\
\hline
\end{tabular}
nistratives nécessaires à la division parcellaire complique le processus et ralentit la mise en œuvre de la requalification.

À ces obstacles techniques et administratifs s'ajoute le manque de ressource financière de la commune. Le condono edilizio n'a sanctionné la pratique de l'abusivismo que par de modestes amendes et les revenus des honoraires versés par les propriétaires privés ne suffisent pas. En outre, une part fixe revient à l'État et la commune ne perçoit que, en moyenne, $7000 €$ par construction abusive à assainir, soit $1 / 3$ du coût effectif de l'intervention qui s'élève à $21000 €$ par construction en moyenne (12). En revanche, cette procédure est un réel avantage pour le propriétaire qui sans cette mise en règle ne peut ni vendre, ni louer, ni léguer son bien immobilier. La somme versée au total par le propriétaire est bien inférieure à la plus-value immobilière induite, ce qui alimente le processus de spéculation foncière.

Le cas de Valle Fiorita, dont la requalification n'a pu être engagée que vingt ans après le vote de la loi sur condono edilizio de 1985, illustre la lenteur de la requalification des zones abusives. Le condono edilizio est loin d'avoir eu tous les effets escomptés: alors que la commune peine à équiper les zones abusives existantes, les constructions spontanées continuent d'envahir la périphérie. En effet, au vu du profit obtenu par les

propriétaires privés grâce au mécanisme de régularisation, le phénomène de l'abusivismo s'est développé dans les années 1990 sous une nouvelle forme, "l'abusivismo de luxe », qui comporte une dimension explicitement spéculative à la différence de l'abusivismo de nécessité de l'après guerre.

\section{Casalotti ou la participation des propriétaires privés: une solution aux lenteurs de la requalification}

Pour régulariser toutes ces nouvelles constructions, deux autres condoni edilizi ont été mis en œuvre en 1994 et 2003 sous les gouvernements Berlusconi. Afin de contourner certains obstacles rencontrés lors du premier condono edilizio, la commune a transformé la procédure initiale. Cette nouvelle procédure autorise la prise en charge des travaux de requalification des zones abusives directement par les propriétaires des biens immobiliers; cette procédure est désignée par le terme d'autorecupero. Au lieu de restituer les honoraires de construction à la commune, les propriétaires les versent dans les caisses d'une « mutuelle » regroupant les propriétaires de la zone urbanisée. À ces propriétaires ex-abusifs, peuvent s'ajouter les propriétaires d'un terrain constructible sur le même « consorzio » (13). La mutuelle gère les fonds à sa disposition. Sur la base $d^{\prime}$ un programme défini par la commune, elle propose les interventions les plus adéquates pour la zone, et les citadins suivent le projet 


\begin{tabular}{|l|c|l|}
\hline \multicolumn{3}{|c|}{ Tableau 2. Projet de Requalification zone toponimi Casalotti Mazzalupo } \\
(230 ha) \\
\hline $\begin{array}{c}\text { Projet de } \\
\text { Requalification }\end{array}$ & $\begin{array}{c}\text { Coûts de la requalification } \\
\text { supportés par le consorzio } \\
\text { (millions d'euros) }\end{array}$ & \multicolumn{1}{|c|}{$\begin{array}{c}\text { Programme de } \\
\text { requalification }\end{array}$} \\
\hline Services primaires & 52,1 & $\begin{array}{l}\text { Réseau d'assainissement, } \\
\text { microstations d'épuration, } \\
\text { éclairage public, voirie et } \\
\text { parking }\end{array}$ \\
\hline Services secondaires & 72,4 & $\begin{array}{l}\text { centre religieux et culturel, } \\
\text { marché, espace vert équipé, } \\
\text { équipement sportif et parc }\end{array}$ \\
\hline Expropriation des sols & 5,2 & $\begin{array}{l}52,6 \text { ha pour destination } \\
\text { publique } \\
29,2 \text { ha destiné à la voierie } \\
\text { publique }\end{array}$ \\
\hline Montant du projet & 129,7 & \\
\hline
\end{tabular}

I'acquisition des terrains nécessaires à l'intégration des nouvelles infrastructures ne passe plus par l'expropriation, mais par des négociations internes aux consor$z i$. La durée des travaux est donc sensiblement réduite et ce montage garantit aux habitants l'investissement des honoraires dus à la municipalité dans leur propre quartier.

En outre, ce mécanisme allège les charges de la commune qui investissait dans la requalification des zones " $\mathrm{O}$ » trois fois plus que le montant les honoraires perçus avec le condono edilizio. En effet, ces dépenses supplémentaires sont désormais supportées soit par des propriétaires ex-abusifs à qui sont offerts, en

et la réalisation des opérations. La mise en place d'un « consorzio di autorecupero » (14) (consortium d'auto-requalification) a pour objectif la réalisation des infrastructures: réseau d'assainissement, station d'épuration, éclairage public, et d'autres équipements publics: écoles, crèches, jardins, parcs et places publiques (15). Cette seconde génération de plans de zone exabusives prend le nom de «toponimi » (16) (toponymie) (Figure 4) et s'inscrit dans le second plan d'assainissement de la périphérie regroupant les constructions abusives développées de 1985 à 1994.

La zone Casalotti-Mazzalupo, située au-delà du périphérique dans le secteur nord ouest de la ville, s'est développée à partir des années 1970 (Figure 3). Ce tissu résidentiel abusif, inclus dans les zones « $\mathrm{O}$ », est requalifié dans les années 1980. Mais au cours de ces années, il continue à s'étendre et atteint aujourd'hui 230 hectares. Cette nouvelle zone d'expansion est incluse dans le second condono edilizio (1994) et le plan de requalification prend la forme des plans de toponimi (Figure 4). Ce mode de requalification offre d'abord une réponse concrète et rapide aux besoins des services primaires. Entre 1994 et 2008, ce sont déjà 130 millions $€$ (17) de travaux d'intérêt public, principalement de réseaux d'assainissement et d'éclairage public, qui ont été réalisés à Casalotti. Le tableau 2 restitue la répartition des dépenses de requalification.

Ce financement direct de la requalification du quartier par les propriétaires simplifie la procédure administrative. De plus, échange de leur contribution plus importante au financement des services, de nouveaux droits de construction sur leur terrain, soit par les honoraires de droits de construction issus de nouvelles opérations privées légales localisées au sein de la zona di toponimi sur des terrains vacants et jusqu'alors inconstructibles. L'augmentation du nombre d'investisseurs pour la requalification conduit à une densification des zones abusives. En densifiant, la commune cherche, d'une part, à canaliser l'étalement urbain et, d'autre part, à rentabiliser les services en réseaux et les équipements.

Les condoni edilizi ont permis d'évaluer l'ampleur de l'étalement urbain, de repérer la localisation des zones d'urbanisation spontanée sur le territoire, d'estimer les besoins en services urbains et de déclencher un processus de requalification. Même si la municipalité romaine reste partagée entre la nécessité d'améliorer l'arrivée des réseaux et le risque de détérioration des sites à valeurs paysagères (Bosi, 2004), craignant l'implantation de nouvelles opérations abusives à proximité des zones nouvellement viabilisées (18), la commune a pu équiper toutes les zones abusives en services primaires de base. Pour les constructions illégales réalisées après le condono edilizio de 2003, la commune a interdit à l'ACEA (Agence communale d'énergie électrique et opérateur du réseau d'eau) d'accepter un contrat de service d'électricité et d'eau potable (19). L'évacuation des eaux est une priorité pour la commune: désormais, tout réseau d'eau requiert la présence d'un réseau d'assainissement aux normes. Toutefois, ceci n'empêche pas le phénomène de 
I'abusivismo de perdurer: les propriétaires agrandissent de manière illégale leur résidence principale (désormais en règle) ou réalisent des nouvelles constructions sur leur terrain à proximité du réseau principal. En ce qui concerne les services secondaires, leur quasi-inexistence en périphérie demeure l'une des préoccupations majeures de la municipalité de Rome. En outre, compte tenu de la faiblesse des transports en commun dans ces zones, le besoin de services en périphérie en vue de limiter les inégalités sociales et d'améliorer la qualité de vie des citadins de ces zones se fait pressant. Mais la réalisation des services secondaires requiert deux éléments essentiels que ne possède pas la commune: des terrains et des ressources financières.

\section{De L'OPPORTUNISME DU SECTEUR PRIVÉ À LA VERTU DE LA NÉGOCIATION PRIVÉ/PUBLIC}

L'urbanisation légale et, corrélativement, l'équipement infrastructurel des zones périurbaines concernées s'inscrivent également, à Rome, dans un contexte spécifique, marqué par la concentration de la propriété du sol aux mains de quelques grands ensembliers urbains (20): grandes familles romaines s'étant diversifiées dans la promotion urbaine (21) pour tirer le meilleur revenu de leurs terres ou promoteurs ayant acquis de vastes réserves foncières. En possession de ressources foncières et financières, ces acteurs contrôlent largement le marché de la production urbaine et constituent donc des interlocuteurs incontournables pour la puissance publique. II convient donc de s'intéresser aux relations entre ces grands ensembliers urbains et la Ville. Les procédures liées à l'urbanisation légale privée seront traitées à partir de deux études de cas: le quartier de La Castellucia, qui illustre les effets de la loi Bucalossi de 1977, et le quartier de Cinquina, qui illustre le dispositif de convenzione urbanistica introduit par la municipalité en 1993, lors du tournant réformiste. Ces deux zones urbaines, situées en extrême périphérie au-delà du GRA (Figure 3) et non desservies par le réseau ferroviaire, sont issues de dynamiques urbaines de constructions privées à des périodes distinctes.

\section{La Castellucia ou l'opportunisme du secteur privé}

En ce qui concerne la réalisation de projets immobiliers privés, une nouvelle procédure de planification est mise en place en 1977 avec la loi Bucalossi (22). Désormais, en Italie, pour chaque transformation ou nouvelle construction sur un territoire communal, les propriétaires doivent participer aux dépenses relatives à l'exécution des opérations via les versements d'ho- noraires de droit à construire. Cette contribution correspond à l'estimation des dépenses de viabilisation du terrain et du coût de construction des équipements et des infrastructures, estimation mise à jours tous les trois ans par l'ISTAT (Institut national de statistique) en fonction de l'évolution du coût de la vie.

Ces honoraires de droit à construire sont calculés en fonction du nombre de $\mathrm{m}^{2}$ et de $\mathrm{m}^{3}$ du projet. Le paiement de ces honoraires permet d'acquérir les droits de construction et les titres de propriété. Une fois les honoraires versés, la commune doit équiper les terrains en réseaux et en équipements publics. Une alternative au versement des honoraires de droit de construction à la commune est proposée dans l'article 11 de cette même loi : celui-ci autorise le propriétaire privé à réaliser directement les opérations d'urbanisation en y investissant les honoraires normalement perçus par la commune.

L'opération urbaine de la Castellucia illustre les enjeux de la mise en œuvre de la loi Bucalossi dans une situation fréquente: celle des faillites frauduleuses. En effet, dans les années 1990, le secteur public est souvent confronté à ce type de faillite. À titre d'exemple, l'entreprise Roma Lazio, l'une des plus grandes entreprises qui opéraient à Rome, en lien étroit avec les grands propriétaires fonciers romains, a vendu de nombreux logements sans arrivée d'eau, de gaz et d'électricité avant de faire faillite. La zone de la Castellucia, située au Nord-Est de Rome, au-delà du périphérique, à l'Est du km 14.5 de la via Cassia et de la ligne ferroviaire Roma/Viterbo, s'étend sur un terrain de 37,2 ha (Figure 3). Son tissu pavillonnaire de faible densité (25 hab./ha) est composé de maisons individuelles ou bi-familiales et accueille 948 habitants. Le projet de lotissement privé débute en 1991. Les travaux de dédoublement de la voie ferroviaire bloquent le chantier pendant trois ans et demi. L'entreprise Cynthia, responsable du projet, fait faillite, alors que les résidences, achetées sur plan, ont été payées par les propriétaires en amont de la réalisation. Le raccordement à certains réseaux (eau et assainissement, gaz et électricité) a été réalisé, ce qui n'est pas le cas des équipements publics, de la voirie, de l'éclairage public, de l'emplacement des containers pour la récolte des déchets et de l'aménagement des espaces verts. Les résidents de la Castellucia subissent directement les répercussions de cette affaire puisque le statut privé du lotissement les rend responsables de la zone. Ils doivent supporter la réalisation des travaux manquants, soit l'équivalent d'un million d'euros. Regroupés en copropriété, les habitants voudraient entamer la réalisation des opérations manquantes, mais le fait que l'affaire 


\begin{tabular}{|c|c|c|c|}
\hline \multicolumn{4}{|c|}{$\begin{array}{l}\text { Tableau 3. Projet de la Castellucia-Loi Bucalossi } \\
\text { ( } 37 \text { ha, } 948 \text { habitants) }\end{array}$} \\
\hline $\begin{array}{c}\text { Projet privé sous Loi } \\
\text { Bucalossi }\end{array}$ & $\begin{array}{l}\text { Coûts définis dans la } \\
\text { convention, pris en } \\
\text { charge par le privé } \\
\text { (millions d'euros) }\end{array}$ & $\begin{array}{l}\text { Programme de } \\
\text { requalification }\end{array}$ & $\begin{array}{c}\text { Montant réellement } \\
\text { dépensé par } \\
\text { l'entreprise privée }\end{array}$ \\
\hline Services primaires & 3,2 & $\begin{array}{c}\text { Réseau d'assainisse- } \\
\text { ment, microstations } \\
\text { d'épuration, éclairage } \\
\text { public, voirie et } \\
\text { parking }\end{array}$ & 2,2 \\
\hline $\begin{array}{l}\text { Services secon- } \\
\text { daires }\end{array}$ & 2,2 & $\begin{array}{l}\text { Centre polyvalent et } \\
\text { gymnase, aménage- } \\
\text { ment d'un espace de } \\
\text { jeu et d'un parc } \\
\text { public }\end{array}$ & 0 \\
\hline $\begin{array}{l}\text { Sol cédé au secteur } \\
\text { public une fois } \\
\text { équipé }\end{array}$ & & $\begin{array}{l}3,6 \text { ha pour destina- } \\
\text { tion publique }\end{array}$ & \\
\hline $\begin{array}{l}\text { Honoraires perçus } \\
\text { par la commune }\end{array}$ & 1,2 & & 0 \\
\hline Montant du projet & 6,6 & & 2,2 \\
\hline
\end{tabular}

dent pas forcément la réalisation des travaux primaires pour construire et I'intégration des réseaux d'infrastructure est rendue plus difficile et plus onéreuse. Deuxièmement, lorsque les promoteurs décident de prendre en charge eux-mêmes la réalisation des opérations «primaires » et « secondaires », ils vendent des lotissements sous-équipés, sans voirie, ni éclairage. L'incapacité de la Ville, d'une part, à investir l'argent perçu dans les quartiers concernés et, d'autre part, à contrôler l'action des promoteurs privés témoigne de la piètre organisation des services municipaux.

\section{Cinquina ou la vertu de la négociation entre le secteur privé et public: la " convenzione urbanistica " (23)}

soit en cours de jugement empêche les derniers acquéreurs de récupérer leurs droits de propriété, nécessaires pour toutes les interventions. La commune, quant à elle, n'a pas perçu les 1,2 M€ que le contractant devait lui reverser pour une partie des honoraires de droit à construire. En outre, les opérations urbaines secondaires prévues à la charge du secteur privé (centre polyvalent et gymnase, espace de jeu et parc public) n'ont pas été réalisées. En devenant propriétaire des 3,6 hectares cédés par le contractant, la municipalité se trouve, une nouvelle fois, dans l'incapacité de réaliser les équipements initialement prévus et d'entretenir ces terrains. Ces espaces vacants ne sont pas sans rappeler le scénario des terrains abandonnés dans les piani di zona des années 1970. Le tableau 3 restitue la répartition des financements des services sur la zone $d^{\prime}$ intervention.

Avec la loi Bucalossi, la commune, en impliquant financièrement le secteur privé dans la viabilisation, ne finance plus directement la rente foncière. Toutefois, cette nouvelle loi présente deux inconvénients majeurs. Premièrement, la commune est confrontée à la difficulté de réinvestir les honoraires de concession dans des délais raisonnables et dans les zones où elles ont été perçues. En conséquence, les promoteurs n'atten-
L'exemple de Cinquina illustre les effets de la Convenzione urbanistica, instaurée par la commune en 1993, en termes de répartition des coûts. À la fin des années 1980, les promoteurs optent systématiquement pour l'article 11 de la loi Bucalossi. Le propriétaire réalise donc directement les opérations urbaines en investissant les honoraires normalement perçus par la commune. La commune se retrouve alors confrontée à un certain nombre de problèmes. Le montant exact des honoraires n'est pas toujours réinvesti comme la loi le prévoit et la commune relève un manque de qualité dans la réalisation des travaux. Pour encadrer les débordements du secteur privé et éviter l'apparition de nouveaux projets sous équipés, la commune instaure donc en 1993 la convenzione urbanistica.

Au titre de cette " convention urbaine », le propriétaire privé devient entièrement responsable du projet, de sa réalisation et du financement des réseaux et des équipements publics. En plus des opérations «primaires » et « secondaires », le propriétaire cède gratuitement à la commune les terrains nécessaires à la viabilisation et aux équipements publics. La convention règle le calendrier et les modalités des procédures de réalisation des équipements publics, le programme de construction des bâtiments et le montant des frais pris en charge par 
I'opérateur. Si le montant des travaux réalisés est supérieur au montant négocié entre le contractant et la commune au titre de la convention, la différence est à la charge du propriétaire. Pour le bon déroulement de la procédure, la commune vérifie la réalisation d'une partie des opérations primaires (réseaux d'eau, d'assainissement et d'énergie) avant de délivrer l'autorisation de construire le projet. Pour obtenir cette autorisation, le propriétaire joint à la convention un document de validation des travaux signé par chacune des entreprises de service de la ville: I'ACEA pour l'eau, I'assainissement et l'électricité et I'ENI (Ente Nazionale Idrocarburi: Entreprise Nationale d'Hydrocarbure) pour le gaz (24). Par la suite, un représentant de la commune valide la fin des travaux seulement si les opérations secondaires et les opérations primaires (éclairage public, voirie) ont été achevées en bonne et due forme. Sans cette validation officielle, la commune ne délivre pas les titres de propriété et le contractant ne peut ni vendre, ni louer, ni léguer son bien (25).

Le projet de lotissement privé Cinquina, inséré dans le nouveau plan régulateur général de 2003, se situe au nord de Rome, au-delà du périphérique (Figure 3). Le terrain de 44,3 ha est composé de 32 immeubles de 5 étages, typologie courante dans les interventions des trente dernières années, et accueille 3103 habitants, soit une densité moyenne de (70 hab./ha). Cette faible densité est le résultat d'une implantation dispersée du bâti sur une vaste parcelle (Figure 5). Le tableau 4 restitue la répartition des investissements dans les services en réseau et services urbains.

La « convention urbaine » simplifie et clarifie nettement la répartition des honoraires de concession et les temps de réalisation des services urbains sont sensiblement réduits. En plus de la garantie de la réalisation en bonne et due forme des opérations primaires et secondaires, le secteur privé a à sa charge deux ans de maintenance à compter de la date de réception. Ces implications obligent les propriétaires à réaliser des travaux de bonne qualité, au risque d'en payer les frais et de ne pas obtenir les titres de propriétés (26).

Les avis sur les apports de cette procédure sont partagés. Certains responsables politiques et certains urbanistes déplorent le fait que le raccordement aux réseaux des nouvelles opérations périphériques ait engendré des dépenses considérables pour la commune. Ils dénoncent spécialement la localisation des zones des grandes opérations privées par rapport aux réseaux existants. Les ingénieurs de l'ACEA, responsables de la

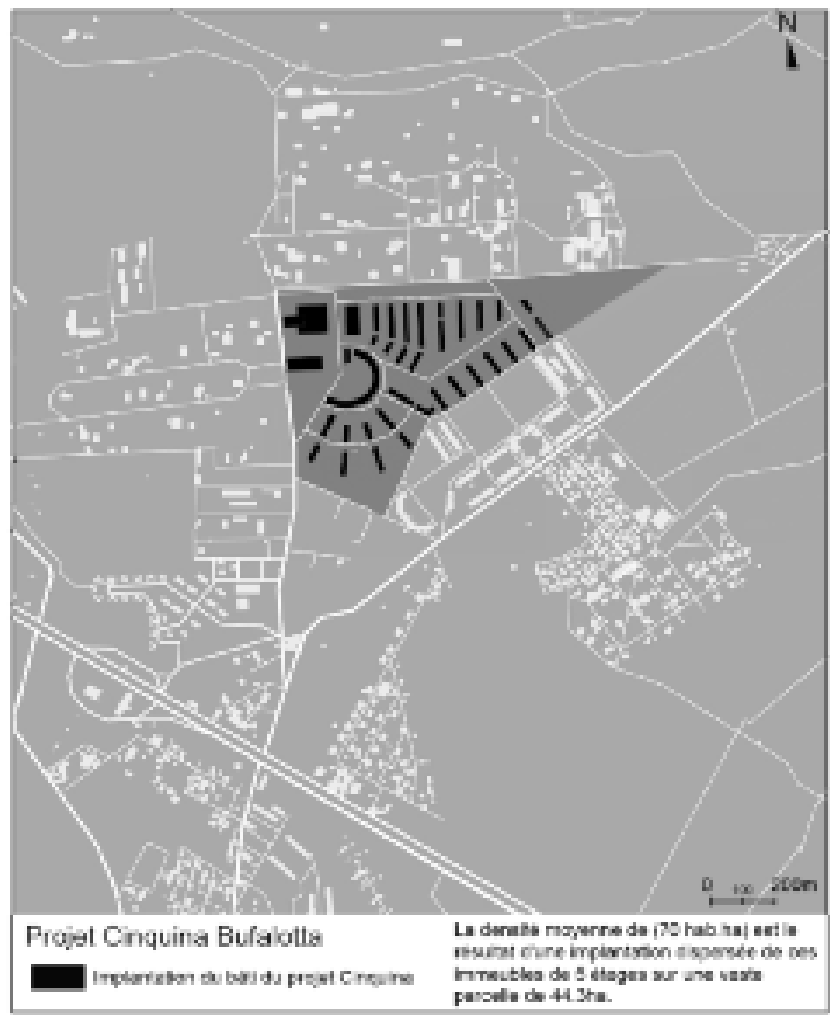

Figure 5. Plan masse du projet Cinquina Bufalotta Source: réalisation de l'auteure

planification des réseaux et de la régulation commerciale, tiennent toutefois à nuancer ce discours (27). En considérant la totalité des projets sur le territoire, ils soutiennent qu'il existe une péréquation: selon les estimations de l'ACEA, le coût prévu de certaines interventions est supérieur au coût réel des travaux, ce qui permet de couvrir tout ou partie du coût de raccordement au réseau existant $d^{\prime}$ 'autres opérations. Par ailleurs, en ce qui concerne infrastructures d'assainissement, la mise en place de systèmes de réseaux décentralisés sur le territoire, bien adaptés aux espaces périphériques de faible densité, avec par exemple la multiplication de petites unités de traitement d'eau, permet d'éviter les surcoûts de prolongation des conduites.

En ce qui concerne les réseaux de transport, à Cinquina, le réseau ferroviaire est inexistant. Seul l'autobus permet d'atteindre le centre de Rome et il faut compter au minimum $1 \mathrm{~h} 20$. Cette situation est loin d'être exceptionnelle et c'est ce qui explique la prédominance des projets privés autour du périphérique lequel permet de rejoindre le centre rapidement en voiture. Le secteur privé aujourd'hui prend à sa charge tous les 


\begin{tabular}{|l|c|l|}
\hline \multicolumn{2}{|c|}{ Tableau 4. Projet Cinquina Bufalotta convenzione urbanistica } \\
(44 ha, 3103 habitants) \\
$\begin{array}{c}\text { Projet privé sous } \\
\text { « convenzione urbanistica » }\end{array}$ & $\begin{array}{c}\text { Coûts définis dans la } \\
\text { convention, pris en charge } \\
\text { par le privé } \\
\text { (millions d'euros) }\end{array}$ & \multicolumn{1}{|c|}{$\begin{array}{c}\text { Programme } \\
\text { de requalification }\end{array}$} \\
\hline Services primaires & 14,1 & $\begin{array}{l}\text { Réseau d'assainissement, } \\
\text { microstations d'épuration, } \\
\text { éclairage public, voirie et } \\
\text { parking }\end{array}$ \\
\hline Services secondaires & 9,7 & $\begin{array}{l}\text { école maternelle, } \\
\text { élémentaire et moyenne, } \\
\text { un parc public de 21 ha, } \\
\text { avec } 9 \text { ha équipés pour un } \\
\text { espace de jeu }\end{array}$ \\
\hline $\begin{array}{l}\text { Sol cédé au secteur public } \\
\text { une fois équipé }\end{array}$ & & $\begin{array}{l}\text { 22 ha pour destination } \\
\text { publique }\end{array}$ \\
\hline Montant du projet & 23,8 & \\
\hline
\end{tabular}

mum d'habitants, la commune est contrainte de desservir des zones urbaines peu denses et très éloignées du centre. Les autobus, quasi vides sur plus de la moitié des trajets, ne sont pas rentabilisés.

Malgré leurs oppositions, réformistes et contre-réformistes s'accordent pour dénoncer la répartition déséquilibrée de la rente urbaine. Certes, à Cinquina, les 24 millions $€$ investis par le secteur privé sont largement compensés par la valorisation foncière du terrain (28), dont la valeur est passée de 6,2 millions $€$ à 55,8 millions $€$, soit un bénéfice d'environ 25,6 millions $€$

réseaux d'infrastructure hormis celui des transports publics. Le coût économique de l'étalement urbain sur les dépenses de la commune se réduit donc essentiellement aux dépenses des transports publics dont le montant financier est nettement supérieur à celui des autres services en réseau. Au-delà d'un mini- (une fois déduit le coût des travaux primaires et secondaires); mais ceci correspond à un partage à peu près égal de la rente entre promoteur et puissance publique. Et Cinquina n'est qu'un exemple parmi d'autres.

L'interprétation de la loi Bucalossi a provoqué d'importantes

\section{Le service de l'eau: un service intégré géré par un unique gestionnaire}

Alors que, dans le domaine de l'énergie, les normes de libéralisation tendent à diviser les branches des services, pour les réseaux d'eau on assiste à l'effet inverse avec la loi Galli, entrée en vigueur en 1996. Les services doivent désormais être gérés par un unique gestionnaire. En contradiction avec les lois européennes, la région Lazio insiste sur sa volonté de non-libéralisation du secteur de l'eau. Les gestionnaires sont responsables d'un territoire délimité par la région: Ambito Territoriale Ottimale (Étendue Territoriale Optimale) et les infrastructures leur sont concédées pour 30 ans mais demeurent propriété du domaine public. Ces territoires institutionnels sont, parfois, non cohérents avec les bassins hydrographiques.

\section{Un réseau d'assainissement décentralisé sur le territoire}

Depuis 1964, le service de distribution de l'eau et de l'assainissement de la ville de Rome est géré par un unique gestionnaire: I'ACEA. En réponse à la loi Galli, ces services sont gérés depuis 2002 par sa filiale ACEA ATO2. A la différence des réseaux d'énergie, le réseau d'assainissement repose sur un système décentralisé. Ce système est constitué de 90 installations avec, d'une part, quatre installations principales correspondant à une division territoriale en quatre bassins géographiques, chacune en mesure de servir une population de 350000 à 1100000 habitants et, d'autre part, une centaine d'installations d'épuration mineures, avec des capacités de 1000 à 50000 habitants, qui desservent des zones urbaines isolées, trop éloignées des installations principales, et qui sont mises en place à l'initiative de l'ACEA mais aux frais des résidents. Pour ces petits réseaux, deux modèles techniques d'assainissement sont retenus: les micros épurateurs et la phytoépuration. Dans le premier modèle, les eaux, une fois traitées à partir de produits chimiques de base, décantent et sont rejetées par des drains enfouis dans la terre. Dans le second modèle, les eaux usées traversent une série de bassins plantés de phragmites et autres plantes d'assainissement et sont ensuite réutilisées pour l'arrosage. Ces nouvelles techniques sont envisagées comme une solution future à grande échelle pour l'approvisionnement des isolats urbains de la périphérie romaine. 
querelles de chapelles opposant les réformistes aux contre-réformistes mais aussi aux écologistes. Ces derniers voient dans cette convention un renoncement de la collectivité à organiser le territoire. Ces opposants dénoncent la faiblesse de la municipalité face aux décisions de programmation induites par les procédures de négociation en faveur du secteur privé et qui se font au détriment des services et des équipements. C'est l'opérateur qui détermine la programmation des équipements publics. Selon les règlements, les fonctions résidentielles et de services devraient se développer proportionnellement mais les opposants font remarquer que, en pratique, ces fonctions se développent de manière inégale, car les terrains destinés aux habitations rapportent plus que ceux destinés aux services (29). La puissance publique ne contrôle donc pas les obligations faites aux promoteurs et montre son incapacité persistante à faire respecter la loi. En outre, les opposants critiquent la localisation des opérations privées qui privilégie la proximité au périphérique.

Toutefois, la convention urbaine est perçue par les techniciens des services en réseau de la municipalité romaine comme une solution à la fois au problème social de la périphérie et au coût de l'étalement urbain (30). La commune règle son problème foncier avec la cession gratuite des terrains et la réalisation des services par le secteur privé. Elle se décharge de toute responsabilité tout en limitant, par un système de garantie juridique, les risques de voir se développer de nouveaux aménagements sans réseaux de services urbains et sans équipements publics. Enfin, il est prévu dans le nouveau plan régulateur de 2006 que la municipalité négocie avec le secteur privé dans le cadre de cette même convention, en plus des honoraires des droits de construction classiques, un financement supplémentaire équivalent aux deux tiers des plus-values résultant du changement de destination des terrains. Ces montants financiers seront investis pour équiper la périphérie en services publics réalisés par le secteur privé. Finalement, la pratique de
Tableau 5. Comparaison de la répartition de la prise en charge des coûts d'infrastructure et d'équipement en pourcentage entre le secteur public et le secteur privé

\begin{tabular}{|c|c|c|c|}
\hline \multirow{2}{*}{$\begin{array}{l}\text { Constructions } \\
\text { légales privées }\end{array}$} & $\begin{array}{c}\text { Castellucia } \\
\text { loi Bucalossi } 1977 \\
\text { (Projet avorté)* }^{*}\end{array}$ & $33,3 *$ & 0 \\
\hline & $\begin{array}{c}\text { Cinquina } \\
\text { convenzione } \\
\text { urbanistica } 1993\end{array}$ & 100 & 0 \\
\hline
\end{tabular}
Prise en charge $\quad$ Prise en charge par le privé en \% par le public en \%

\begin{tabular}{c|c|c} 
Valle fiorita & & \\
condono edilizio & 6,3 & 93,7
\end{tabular}

\begin{tabular}{l|l}
\hline ono edilizio & 6,3 \\
\hline
\end{tabular}

Casalotti

torecupero 1994

* Le projet de la Castellucia a été avorté suite à la faillite du secteur privé. Cette situation
explique la prise en charge partielle par le secteur privé du montant du projet (seulement cé initialement dans sa totalité. la négociation, à travers la convention urbaine, semble apporter un progrès très net par rapport à la situation qui prévalait auparavant. Ce financement des services urbains en périphérie permet de surmonter les contraintes liées à l'expropriation: coûts élevés et caducité au terme de 5 ans. On constate que la commune n'a fait état que de peu de revendications et d'oppositions lors de la période du gouvernement de centre gauche quant au choix d'implantation et de programmation du secteur privé. Ceci témoigne, pour les opposants, de ce que la commune s'est pliée aux exigences des grands propriétaires privés. Mais cela peut aussi signifier qu'elle ne sort pas entièrement perdante de cette négociation. En effet, Cinquina se situe à proximité du piano di zona Cinquina des années 1970: ainsi, grâce aux équipements réalisés et financés par le secteur privé, la commune est enfin parvenue, quarante ans après l'apparition de ces projets publics non aboutis, à améliorer les conditions de vie dans ces quartiers.

\section{Conclusion}

L'étalement urbain, avec son cortège d'enjeux sociaux, sanitaires et environnementaux, pèse considérablement sur les finances de la commune de Rome depuis désormais plus de trente ans, ce qui explique le discours alarmiste de la municipalité de Rome. L'impact de l'étalement urbain sur les coûts des réseaux d'infrastructure y est aggravé par l'abusivismo et, à un degré moindre depuis la loi Bucalossi, par les stratégies spa- 
tiales/foncières des promoteurs. Les surcoûts les plus importants concernent les services nécessitant des infrastructures lourdes, notamment les transports publics, la voirie, la distribution d'eau et le traitement des eaux usées.

La mise en place des nouvelles procédures analysées dans cet article montre une volonté de la part de la commune de financer les opérations de requalification de la périphérie. Les moyens utilisés sont divers et témoignent de pragmatisme et de capacité $d$ 'innovation puisqu'ils font appel à l'intervention du secteur privé dans le déploiement des réseaux. Ces mécanismes de financement limitent les frais de la commune et lui évitent toute prise de risque. Les coûts de raccordement aux réseaux principaux sont supportés par les promoteurs ou par les résidents mettant en commun leurs honoraires de droits de construction.

Alors que la commune a pu être présentée comme victime d'un jeu de négociation qui lui échappait totalement en ce qui concerne la coordination transport/urbanisme (Nessi, Delpirou, 2009), une analyse de la construction des réseaux en situation post-abusivismo et de l'évolution des procédures de planification montre que la commune tire un certain avantage de ces négociations avec le secteur privé. Face à la fatalité historique, au lourd héritage lié au poids du secteur privé dans les dynamiques urbaines, la commune n'a toujours pas les moyens législatifs pour réglementer et limiter l'usage des sols. Son unique marge de manœuvre se situe donc dans sa négociation avec le privé. Dès lors que le contrôle public des sols et de leur usage est impossible, la mise en place de négociations entre acteurs publics et privés pour le financement des réseaux $d^{\prime} i n-$ frastructure de service se présente comme une solution relativement effective et efficace pour le développement coordonné des réseaux, des services et de l'aménagement. Même si la commune paraît soumise aux pressions privées, les techniciens affirment qu'elle y trouve certains avantages. Si, face à sa pénurie de réserve foncière et à son manque de moyens financiers, la commune s'avère incapable d'équiper les zones ex-abusives et héritées des piani di zona, en revanche, lors de la négociation avec le secteur privé, elle peut orienter des interventions et faire financer des services en échange de nouveaux droits à construire. Le fait que ces nouvelles opérations privées soient systématiquement localisées à proximité des zones sous-équipées en services urbains peut être lu comme le résultat d'une stratégie de la commune pour équiper la périphérie aux frais du secteur privé. Finalement, la municipalité romaine constitue un protagoniste à part entière de ce jeu de négociation, bénéficiant essentiellement de deux avantages: I'allégement de ses charges et la réduction (Tableau 5), par une requalification progressive de la périphérie, des déficiences des politiques urbaines antérieures résultant de la non-maîtrise du foncier. Même si cela suppose pour la Ville d'accepter certains compromis, qui lui sont reprochés par les anti-réformistes, les négociations avec le privé lui procurent un certain pouvoir et une certaine marge de manœuvre, qu'elle ne possédait pas auparavant, en ce qui concerne la maîtrise du foncier et la répartition de la rente urbaine $d^{\prime}$ une manière moins inégalitaire.

Hélène Nessi est Architecte-Urbaniste, chargée d'étude à 6T-Bureau de recherche et doctorante en Aménagement du territoire au sein du Latts (Laboratoire Techniques Territoires et Sociétés). Elle mène sa thèse sur les incidences du cadre de vie sur la mobilité de loisir et à longue distance à Paris et à Rome, "l'effet barbecue ". nessi.h@gmail.com

* La recherche dont est issu cet article a bénéficié du soutien de I'Agence Nationale de la Recherche (projet $n^{\circ}$ ANR-05BLAN-0344)

\section{NOTES}

(1) En vertu de l'article 5 bis de la loi 359/92, la valeur d'expropriation d'un terrain n'est que la moitié de sa valeur de marché. Mais il s'agit d'une valeur que l'administration municipale ne peut souvent pas supporter, surtout lorsqu'elle s'applique à de grandes propriétés. Sur la crise de l'expropriation en Italie: cf. Oliva, 1998.

(2) Au cours de son XXIe Congrès de Bologne, à la fin de 1995, I'INU définit de nouveaux principes et de nouvelles règles d'urbanisation, en vue d'une nouvelle réforme de la loi d'urbanisme. La majorité de Centre Gauche, qui paraissait engagée dans la nouvelle Réforme de l'urbanisme, a laissé passer ses cinq années de mandat, sans la mener à bien. Cette réforme n'a finalement pas été acceptée par le Parlement.

(3) « II nuovo piano regolatore e Roma. I dati dello svillupo », Commune di Roma, 2003

(4) Les opérations primaires sont les infrastructures qui permettent de fournir des services au domicile des particuliers. II s'agit principalement de la voirie, des réseaux de distribution d'eau alimentaire, d'électricité, de gaz, de communication de l'information, ainsi que l'éclairage public et les divers équipe- 
ments liés à la collecte et au traitement des eaux usées.

(5) Les opérations secondaires sont des interventions d'intérêts publics. Ces superstructures correspondent aux lieux de consommation collective ouverts au public: les écoles, les piscines, les crèches, les parcs et les places, les centres culturels et sportifs, les maisons de retraire et maison de quartier, etc.

(6) Le terme désigne la période la plus contemporaine de la ville, qui s'ouvre avec la proclamation de Rome comme capitale du nouveau Royaume d'Italie en 1870.

(7) Cf. Alberto Caracciolo, 1956 et Italo Insolera, 1962.

(8) Les piani di zona sont des secteurs d'expropriation pour cause d'utilité publique (constructions de logements publics à bas prix). Issus de la loi 167 , ils s'inscrivent dans le plan régulateur de 1962.

(9) Le plan régulateur est composé de différentes zones aux destinations diverses, chaque type de destination est référencé par une lettre de l'alphabet. La lettre « $O$ » renvoie à des aires urbaines délimitées issues d'un développement urbain illégal.

(10) Jusqu'au début des années 1970, les résidents des quartiers abusifs se débrouillent pour se raccorder aux réseaux d'électricité et d'eau potable, pour certains de manière illégale et étonnamment pour d'autres de manière légale. En effet, face à un niveau de précarité et à de terribles conditions sociales et sanitaires, la commune autorise les entreprises de services en réseau à établir des contrats avec les habitants. Par la suite la municipalité met en place quatre phases successives de requalification des zones abusives existantes avec, en 1974, un piano di risanamento idro-sanitario concernant la réalisation des réseaux d'eau et d'assainissement; en 1976, un plan de construction d'équipement scolaire; en 1979, un plan d'éclairage public et en 1980, un plan d'extension du réseau de gaz. Enfin, dans le cadre du premier condono edilizio, la requalification des zones abusives concerne la réalisation et I'amélioration du réseau d'assainissement, de la voirie et de l'éclairage public.

(11) Interview de S. Bottoni, représentant à l'urbanisme municipio XIII, Commune de Rome, avril 2006.

(12) Interviews M. Veronesi, Responsable de Legambiente, avril 2006 et avril 2007.

(13) Le consorzio est une entité géographique délimitée par la commune, regroupant un certain nombre d'habitants.

(14) Le consorzio di autorecupero regroupe dans une mutuelle les honoraires de concession des résidents d'une identité géographique pour réaliser les travaux de requalification de la zone.

(15) Interview de I'Ingénieur Martinelli et de l'Arch. G. Mandarelli, Recupero della periferia -Direzione del dipartimento XIX - Sviluppo e recupero della periferia, Commune de Rome, avril 2007.

(16) Ce terme, réapproprié par les élus municipaux romains, définit initialement la science des noms de lieux, hérités de leur particularité: patrimoine architecturaux, caractéristique géographique (cours d'eau, dénivelé) ou présence d'un personnage singulier.

(17) Interview de I'Arch. M. di Giovine, Responsable de la direction du Département XIX-Sviluppo e recupero della periferia, Commune de Rome, avril 2007.

(18) Interview de S. Bottoni, représentant à I'urbanisme municipio XIII, Commune de Rome, avril 2006.

(19) Interview de G.Panunzi, Départ. XI, Ufficio condono Edilizio e sistema informativo per il territorio, Commune de Rome, avril 2007.

(20) Certains promoteurs, comme Caltagirone, parviennent même à financer entièrement leur projet sans avoir recours à d'autres investisseurs. Le groupe Caltagirone, dirigé par trois frères issus d'une grande famille de la bourgeoisie commerçante romaine. Regroupant un ensemble de sociétés opérant principalement dans les secteurs de l'immobilier, de l'industrie du ciment et du BTP, il s'est diversifié en entrant dans les assurances et les médias ( II Messaggero ») et les télécommunications. Considéré comme étant I'un des plus puissants promoteurs de la péninsule, Caltagirone est au cœur de nombreuses polémiques, en particulier pour ses activités spéculatives et ses liens opaques avec la classe politique.

(21) Maître de l'ensemble du processus de production circonscrite au marché de la construction, ces acteurs privés élargissent aussi leurs activités avec la conception, l'ingénierie, la prestation des services à la maîtrise d'ouvrage, la promotion immobilière et la gestion du patrimoine immobilier, la gestion opérationnelle et administrative des complexes commerciaux, tertiaires et des hôtels.

(22) Legge 28 janvier 1977, n¹0- (Bucalossi)- (G.U.29-119977, N. 27). "Norme per l'edificabilità dei suoli. »

(23) La convention réunit les plans et toutes les données prescriptives et économiques du projet.

(24) Interview de E. Zendri, Gruppo ACEA, Distribuzione Spa-Pianificazione reti-Regolazione, nov. 2006.

(25) Interview de G. Schettini, Département VI -Politiche di Attualizzazione, Lottizzazione e Convenzione, Commune de Rome, nov. 2006 et avril 2007.

(26) Cf. note 29.

(27) Le coût du projet se réfère à un calcul conventionnel, institué par la loi italienne. Il définit le coût de la fourniture d'un service à partir de deux montants: I'un dépend de la distance en mètres entre le réseau existant et le nouveau point où l'on doit acheminer le réseau, et l'autre correspond à la quantité d'énergie ou d'eau demandée en fonction du projet. Les résultats sont souvent éloignés du coût réel, aussi bien de manière positive que négative.

(28) La valeur au $\mathrm{m}^{2}$ passe en moyenne de $20 €$ à $180 €$ (Source: Italia Nostra, Legambiente et WWF). 
(29) Le régime immobilier actuel présente un inégal développement entre habitations et services, deux fonctions de la ville qui devraient pourtant se développer proportionnellement, car la rente foncière urbaine rapporte plus sur les terrains destinés à l'habitation que sur ceux destinés aux services. Les zones destinées aux services ne permettent par de grands bénéfices, parce qu'elles n'ont qu'un seul acquéreur, la collectivité. Les institutions publiques ne tirent donc pas d'intérêts financiers de l'usage des sols, mais doivent au contraire financer la construction du service et ensuite sa gestion. L'autorité publique n'est donc pas en condition de payer des prix élevés pour acquérir le terrain.

(30) Marco F. Lazzara, Assesseur municipio XIX de la Commune de Rome, novembre 2006.

\section{BibliograPHIE}

Alonso W, 1968, Location and Land Use, Towards a General Theory of Land Rent, Cambridge, Harvard University Press

BARBieri C.-A., Oliva F., 1995, « Le prospettive perequative per un nuovo regime immobiliare ", Urbanistica Quaderni, 7, pp. 144-157.

BARBIERI C.-A., 1998, " La perequazione urbanistica: quattro ragioni per la riforma », Urbanistica informazioni, $\mathrm{n}^{\circ} 157$, pp. 4-5.

BERDINI P., 2006, "La cancellazione della campagna romana », in: "No sprawl », M-C. Gibelli e E.Salzano, Alinez Editrice, pp. 201-215.

Bosı R., 2003, Il campionario degli artifici escogitati per legittimare gli abusi commessi dentro il parco di Veio, Verdi Ambiente e società

Bosı R., 2004, Riferimenti normativi in materia di repressione degli abusi edilizi, Verdi Ambiente e società.

CAmagni R., 1992, Economia urbana. Principi e modelli teorici, Nis, Roma

Caracciolo A., 1956, Roma Capitale. Dal Risorgimento alla crisi dello Stato liberale, Rome, Riuniti (réed. 1993)

Cомву J., 2003, "La formation de la valeur sur les six marchés fonciers », Études Foncières, n¹01, pp. 18-23.

Della Seta P., Della Seta R., 1988, I suoli di Roma. Uso e abuso del territorio nei cento anni della capitale, Editori Riuniti, Biblioteca di storia

Delpirou A, 2009, La fin de la ville loin du fer? Transport et urbanisation dans la Rome contemporaine: les politiques publiques face aux héritages territoriaux, Thèse de doctorat en géographie, Nanterre, Université Paris Ouest - Nanterre La Défense, sous la direction de C. Vallat.

Franck R., 1992, Microeconomia: Comportamento razionale, market, istituzioni, Milano: Mc Graw-Hill

FusCO GIRARD L. (ed.), 1997, "La perequazione urbanistica: le esperienze e le questioni », Urbanistica, n¹09, pp. 51-54.

GarCIA-Bellido J., 1997, "Open inflation models and gravitational wave anisotropies in the $\mathrm{CMB}^{\prime \prime}$, Phys. Rev. $D, \mathrm{n}^{\circ} 56$, pp. 3225-3237.
Gibellı M-C., 2003, « Flessibilità e regole nella pianificazione strategica: buone pratiche alla prova in ambito internazionale », in: Pianificazione strategica per la città: riflessioni dalle pratiche, A.Spaziante, T.Pugliese, Milano, Franco Angeli pp. 53-78.

GiBelLi M-C, 2006, "La dispersione urbana costi collettivi e riposte normative », in: No sprawl, M-C. Gibelli e E.Salzano, Alinez Editrice, pp.79-111.

GEORGE H., 1880, Progrès et pauvreté : enquête sur la cause des crises industrielles et de l'accroissement de la misère au milieu de l'accroissement de la richesse: le remède, trad. PL Le Monnier, 2nd éd. de 1925, New-York, R.Scalkenbach

HaGMAN D. et MISCZYNSKI D. (eds.), 1978, Windfalls for Wipeouts, Planners Press, Chicago

INSOLERA I., 1962, Roma moderna: un secolo di storia urbanistica, Turin, Einaudi

Jaglin S., Boucher-Hedenström F., Pflieger G., Rutherford J., VAuCELLE S., 2008, Étalement urbain et services en réseaux. Réflexions exploratoires dans quatre villes moyennes européennes: Bordeaux, Nantes, Lausanne, Stockholm, Ministère de l'Ecologie, de l'énergie, du développement durable et de l'aménagement du territoire

JACOBS H., 1997, « Programmi di trasferimento dei diritti edificatori in USA: oggi e domani », Urbanistica, n¹09, pp. 62-64.

JOHNSTON R. et MADISON M., 1997, "From Landmarks to Landscapes", Journal of the American Planning Association, vol. 63, n³, pp. 365-3

LANOtTE, H., Rossi, D., 1995, "Négocier les droits sur le sol », Études Foncières, ${ }^{\circ}{ }^{68}$, pp. 19-26.

LÉNINE V-I., 1907, Programme agraire de la sociale démocratie dans la première révolution russe de 1905-1907, Moscou, Éditions du progrès, 1967

Lenzinı R., Ruggero G., 2004, Condono Edilizio, Guida Pratica e normativa, Legislazione Tecnica

LIPIETZ A., 1974, Le tribut foncier urbain: circulation du capital et propriété foncière dans la production du cadre bâti, Paris, Maspero 
Malthus T-R, 1820, Principes d'économie politique considérés sous le rapport de leur application pratique, Paris, CalmanLévy, 1969

Marshall A., 1890, Principles of Economics, New-York: Macmillan, 1949, London, Macmillan, 8th ed., 1998

MArx K., 1894, Le capital, critique de l'économie politique, livre premier: le développement de la production capitaliste, tome 3, Paris, Éditions Sociales, trad. C. Cohen-Sélal et G. Badia, 1974-78 (Das Kapital:Kritik der politischen Okonomie. Der Produktionsprozee das Kapital. Der globale prozess des Wirtschaftlichen Produktion.)

Mıcelu E., 1999, "La négociation des droits à bâtir en Italie », Études Foncières, n82, pp. 17-22.

Micell E., 2002, "Development Rights Markets to Manage Urban Plans in Italy", Urban Studies, vol. 39, n¹, pp. 141154.

MiCelLı E., 2003, « La perequazione e il trasferimento dei diritti edificatori per la gestione innovativa dei piani », in Archivio di Studi Urbani e Regionali, Franco Angeli, anno XXXIV no77, pp. 151-166.

MILL J-S, 1848, Principes d'économie politique avec quelques unes de leurs applications à l'économie sociale, trad. $\mathrm{H}$. Dussard et de D. Courcelle-Seneuil, 3ème édition, 2 tomes, Paris, Guillaumin, 1873

Musolino D., Guerzonı M., 2003, "Città dispersa e costi collettivi: il caso di Bologna ", Scienze Regionali, nº1, pp. 107-112.

Oliva F., 1998, "La crisi dell'esproprio », Urbanistica Informazioni, n¹57, pp. 36-37.

POMPE S., 1997, "Cinque nodi lungo la via della perequazione in Italia », Urbanistica, n¹09, pp. 71-74.

POMPE S., 1998, II piano regolatore perequativo, Hoepli, Milano

ProudHOU P-J., 1840, Qu'est ce que la propriété? ou recherche sur le principe du droit et du gouvernement, premier mémoire, Chronologie et introduction de James E., GarnierFlammarion, 196

Pruetz R., 1996, Putting Transfer of Development Rights to Work in California, Solano Press Books, Point Arena CA
ReNARD V., 1998, L'utilisation des permis négociables dans le domaine de la gestion des sols, Atelier sur les systèmes de permis négociables nationaux pour la gestion de l'environnement: questions et défis. Direction de l'environnement, OCDE, Paris

Renard V., 2007, "Property Rights and the Transfer of Development Rights. Questions of Efficiency and Equity", Town Planning Review, vol. 78, n¹, pp. 41-60.

Ricardo D., 1817, Des principes de l'économie politique et de l'impôt, trad. C. Soudan à partir de l'édition anglaise de 1821 utilisée par Sraffa P., présentation de F-R. Mathieu, Paris, Flammarion, 1992

Rossı A., 1975, "Il problema della periferia nella città moderna », Scritti sull'architettura e la città 1956-72.

Santuccio C., Giancola F., Picciotto M., 1976, Abusivismo per necessità, Roma, Kappa

StanGhellini S. (ed.), 1993, Per la riforma urbanistica del regime immobiliare, Urbanistica Quaderni, $\mathrm{n}^{\circ} 13$.

TOPAlov C., 1973, Capital et propriété foncière. Introduction à l'étude des politiques foncières urbaines, Paris, Centre de Sociologie Urbaine

Turner R. K., Pearce D., Batemani I., 1996, Economia Ambientale, Bologna: Il Mulino

VeNUTI G., 1991, L'urbanistica riformista. Antologia di scritti, lezioni e piani a cura de Oliva F., Etaslibri.

VENUTI G., 2003, « La rente urbaine » in: L'urbanisme réformiste, trad. S. Lana à partir de l'édition italienne de 1991, p. 42.

VENUTI G., 2003, "Il piano regolatore di Roma: scongiurare la contro riforma urbanistica ", Urbanistica Informazioni, n¹87, pp. 82-85.

Von ThüNEN J-H., 1826, Von Thünen's Isolated State, translated by C.M. Watenberd, intro de P. Hall, Oxford, Pergamon Press, 1966 (Der Isolierte Staat).

Walras L., 1860, L'économie politique et la justice, éd. Dockès P., P-H. Goutte, Hébert C. et al, Paris, Economia, 2001

WALRAS L., 1896, "Théorie de la propriété », Revue socialiste, tome 23, $\mathrm{n}^{\circ} 138, \mathrm{pp} .668-681$, juin et tome $24, \mathrm{n}^{\circ} 139$, pp.23-35, juillet. 